\title{
Real contact ratio and tooth bending stress calculation for plastic/plastic and plastic/steel spur gears
}

\author{
Toni Jabbour ${ }^{1, *}$, Ghazi Asmar ${ }^{2}$, Mohamad Abdulwahab ${ }^{3}$, and Jose Nasr ${ }^{1}$ \\ ${ }^{1}$ Department of Mechanical Engineering, ISAE-CNAM Lebanon, P.O. Box: 1136175 Beirut, Lebanon \\ 2 Department of Mechanical Engineering, Notre Dame University, Zouk Mosbeh, Lebanon \\ ${ }^{3}$ Department of Mechanical Engineering, Faculty of Engineering 1' Lebanese University, Tripoli, Lebanon
}

Received: 16 July 2020 / Accepted: 29 March 2021

\begin{abstract}
This paper presents an iterative method for calculating the effective contact ratio and the bending tooth stress for a pair of plastic/plastic and plastic/steel spur gears with an involute profile. In this method, the pinion and the gear are modeled, at each moment of the mesh cycle, as equivalent springs in parallel undergoing the same displacement along the line of action. This leads to the calculation of the bending stress by taking into account the number of teeth initially in contact and those which enter in contact prematurely. We also investigate the influence of certain gear parameters, such as, the number of teeth, the pressure angle, and the module on the behavior of a pair of meshed gears. In addition, the variation of the bending stress at the tooth fillet is investigated for a pair of plastic/plastic and a pair of plastic/steel spur gears, in order to determine the critical configurations for which the bending stress is maximum. In general, the results obtained from the present method also show that the stress variation in plastic/plastic gears differs markedly from that in plastic/steel gears.
\end{abstract}

Keywords: Plastic gears / spur gears / contact ratio / finite element modeling / bending stress

\section{Introduction}

Plastic gears are commonly used in many applications, such as, in motor vehicles, office machines, home appliances, and other pertinent systems and devices. However, the fundamental knowledge of the real behavior of plastic spur gears under loading does not seem to have kept pace with the increasing number of gear applications. Gear teeth are deformed under load, causing the real contact ratio of loaded gears to be higher than the theoretical contact ratio used to model stress conditions according to ISO 6336 [1].

Among the earlier research undertaken on the behavior of plastic gears, one finds the works by Hall et al. [2] and Cornelius et al. [3]. In these works, high speed photography of an acetal/acetal gear pair transmitting load, and measurements of contact ratios show that several teeth carry the load at all times. Based on this, Yelle [4] developed a method for the design of thermoplastic gears. A recent literature review shows a keen interest in the study of the structural analysis of plastic/plastic spur gears [5-12]. This is an addition to the development of analytical-

\footnotetext{
* e-mail: tjabbour@isae.edu.lb
}

iterative methods [13-15] to calculate the tooth root stress of plastic spur gears meshed with steel gears by taking the real contact ratio into consideration.

The objective of this paper is to predict the behavior of plastic spur gears under loading. To this end, we propose a method to calculate the real contact ratio and tooth bending stress of a pair of plastic/plastic and plastic/steel gears subjected to an applied torque $T$. The results obtained from the suggested method are validated by the finite element method (FEM). The effects of the gear parameters, on the contact ratio and on the tooth root stress, are then investigated.

\section{Mechanics of a pair of loaded plastic gears}

For plastic gears, the materials are not rigid. Consequently, the teeth will deform under the applied load. Consider for example Figure 1 which shows teeth $i_{1}$ and $i_{2}$ in contact and teeth $k_{1}$ and $k_{2}$ about to make contact. The torque applied on the driving gear will cause it to rotate an angle $\Delta \theta$ due to deformation of teeth $i_{1}$ and $i_{2}$. At a certain point of engagement, the deformation of tooth pair $i_{1} i_{2}$ will be sufficient to cause premature contact of tooth pair $q_{1} q_{2}$, before the ideal engagement at point $A$, and of teeth pair 


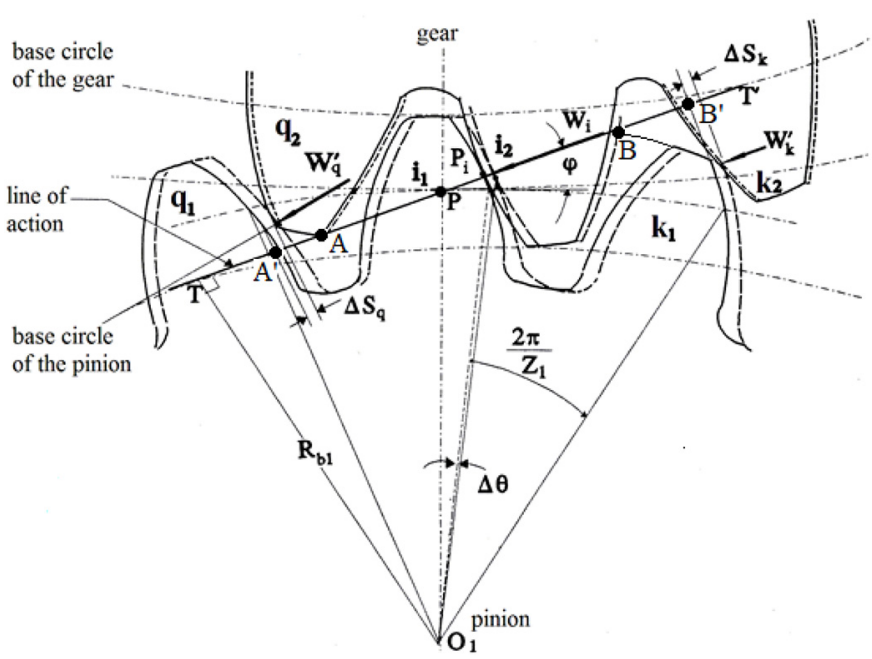

Fig. 1. Deformations of the profiles of the teeth under the effect of the applied torque.

$k_{1} k_{2}$, after the ideal engagement at point $B$. This situation is represented by the dotted lines in Figure 1.

Since each tooth pair can be replaced by a pair of springs in series (of compliance $w_{1}+w_{2}$ ), the pinion and the gear could be modeled as equivalent springs in parallel undergoing the same displacement $R_{b 1} \Delta \theta$ along the line of action [16]. If this displacement is greater than the separation distance $\Delta S_{q}$, then the tooth pair $q_{1} q_{2}$ could enter in contact prematurely (see Fig. 1). If this displacement is greater than $\Delta S_{k}$ then the tooth pair $k_{1} k_{2}$ could also enter in contact. Consequently, with plastic gears, it is possible for several pairs of teeth to enter in contact prematurely.

To calculate the real contact ratio, one must determine, at every position of the pinion and the gear during the meshing cycle, the value of the angle of twist $\Delta \theta$, to compare the value of $R_{b 1} \Delta \theta$ with the distances separating the tooth pairs not in contact. This gives the positions of points $A^{\prime}$ (beginning of contact) and $B^{\prime}$ (end of contact) along the line of action.

\section{Calculation method}

To gain a better appreciation of the problem, let us consider the case of a spur pinion meshing with a gear.

Let $\Delta \theta$ be the rotation of the pinion produced by the applied torque $T$ (see Fig. 1). This results in a deformation of the teeth which are in contact between the pinion and the gear.

It has been shown by Jabbour and Asmar [16], that the deflection of a point $P$ of the teeth in contact, is constant along the line of action which is also the direction of the transverse load $W_{t r}$. The value of this deflection is given by $R_{b 1} \Delta \theta$. Two cases are considered

- The load carried by a pair of teeth $i$ which are initially in contact is

$$
W_{t r i}=\frac{R_{b 1} \Delta \theta}{w_{i}}
$$

where $w_{i}$ is the compliance of the pair of teeth in contact. This compliance varies according to the position of the point of contact on the line of action.

- The load carried by tooth pair $k_{1} k_{2}$ which enter in contact prematurely is

$$
W_{t r k}^{\prime}=\frac{R_{b 1} \Delta \theta-\Delta S_{k}}{w_{k}}
$$

where $\Delta S_{k}$ is the separation distance of the tooth pair $k_{1} k_{2}$ before they enter in contact prematurely.

For the moment, assume that the values of $\Delta S$ and the compliance $w$ can be calculated for various engagement positions of a particular gear pair.

Since the sum of all torques is equal to the total applied torque $T$, we can write

$$
T=\sum_{i=1}^{n_{1}} T_{i}+\sum_{k=1}^{n_{2}} T_{k}=\sum_{i=1}^{n_{1}} W_{t r i} R_{b 1}+\sum_{k=1}^{n_{2}} W_{t r k}^{\prime} R_{b 1 k}^{\prime}
$$

where $n_{1}$ and $n_{2}$ are, respectively, the number of teeth initially in contact and those which become prematurely in contact, and $R_{b 1 k}^{\prime}$ is an equivalent radius from which the torque of the load $W_{t r k}^{\prime}$, with respect to the pinion center, is obtained. The method of calculation of $R_{b 1 k}^{\prime}$ is given in the appendix.

Now from equations (1) through (3), we can relate the twisting angle $\Delta \theta$ to the total torque $T$ as follows

$$
\Delta \theta=\frac{T+\sum_{k=1}^{n_{2}} \frac{R_{b 1 k}^{\prime} \Delta S_{k}}{w_{k}}}{R_{b 1}^{2}\left(\sum_{i=1}^{n_{1}} \frac{1}{w_{i}}+\sum_{k=1}^{n_{2}} \frac{R_{b 1 k}^{\prime}}{R_{b 1} w_{k}}\right)}
$$

Equation (4) enables one to determine the distribution of the load on each line which is initially in contact. Combining equations (1) and (4), and taking into account that the total transmitted load between the pair of gears is $W_{\text {tot }}=\frac{T}{R_{b 1}}$, we get

$$
W_{t r i}=\frac{W_{t o t}+\sum_{k=1}^{n_{2}} \frac{R_{b 1 k}^{\prime} \Delta S_{k}}{R_{b 1} w_{k}}}{\sum_{i=1}^{n_{1}} \frac{1}{w_{i}}+\sum_{k=1}^{n_{2}} \frac{R_{b 1 k}^{\prime}}{R_{b 1} w_{k}}} \frac{1}{w_{i}}
$$

The procedure to determine the real contact ratio is to assume, first, that there are no other teeth in contact except those which were in contact initially, i.e. $n_{2}=0$. Equation (4) gives the value of $\Delta \theta$ resulting from the deflection of teeth. This value of $R_{b 1} \Delta \theta$ must then be compared with the separation distance $\Delta S$ of the pairs of teeth just before and just behind the teeth initially in contact. If $R_{b 1} \Delta \theta$ exceeds either or both of these values, we conclude that these pairs of teeth are likely to be in contact, i.e. $n_{2}$ is equal to 1 or 2 . Therefore, equation (4) must be solved again using the compliances of the pairs of teeth which enter in contact prematurely, and the new value of 

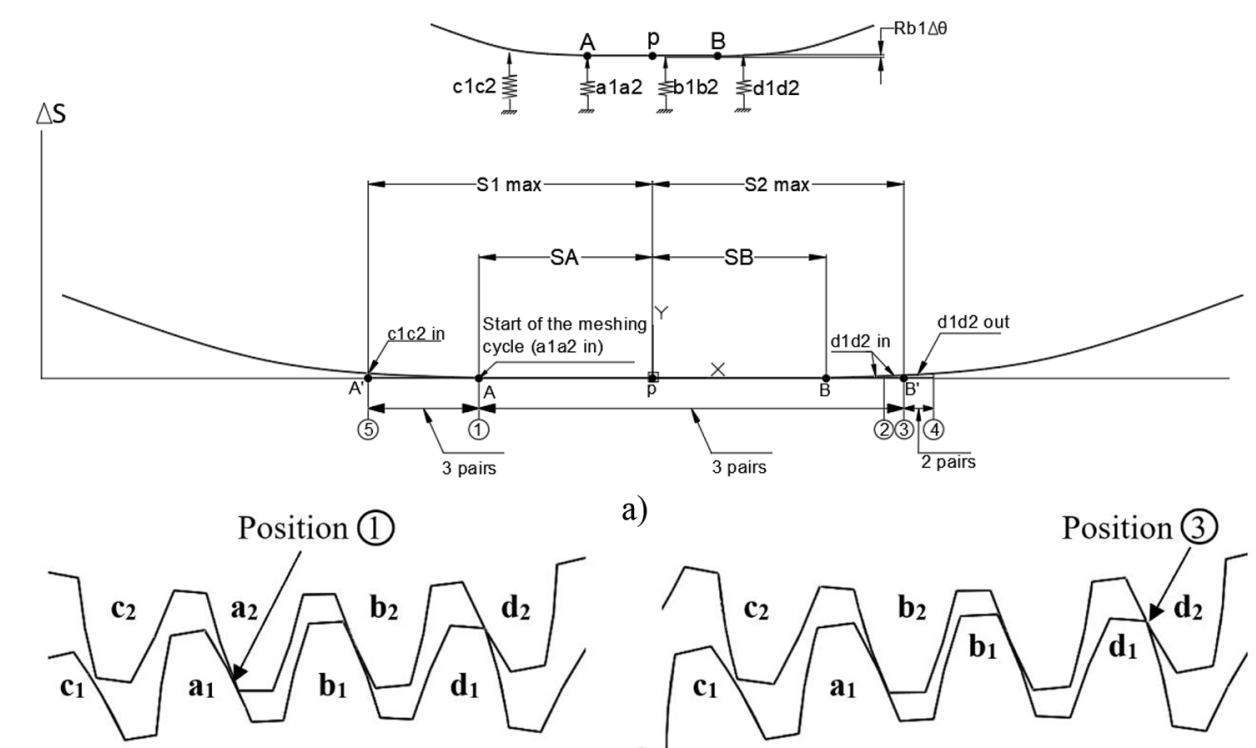

a)

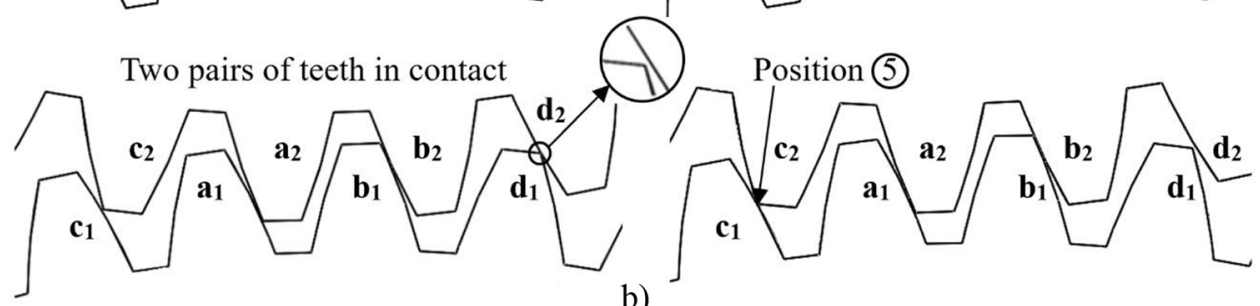

b)

Fig. 2. Behavior of a Nylon 66 pair of plastic gears with 60 teeth each with an applied torque of $400 \mathrm{Nm}$.

$R_{b 1} \Delta \theta$ is obtained. If this new value of $R_{b 1} \Delta \theta$ exceeds the values of the separation distance of the teeth which are just before of and behind the teeth which became in contact prematurely, the procedure must be repeated by increasing the value of $n_{2}$ by 1 or 2 . The process stops when it becomes no longer possible to find teeth which enter in contact prematurely. In this case, we conclude that for this value of $\Delta \theta$, no new teeth will be in contact.

Once the value of $\Delta \theta$ is obtained, we calculate the loads at each point of contact using equations (2) and (5). In this way, the load and stress distributions on the initial lines of contact can be determined, as well as the total number of contact teeth on the pair of gears.

The results of such calculation can be summarized as shown in Figure 2. This considers the behavior of a pair of $20^{\circ}$ pressure angle Nylon 66 plastic gears with 60 teeth each $\left(Z_{1}=Z_{2}=60\right)$ with a torque of 400 N.m. The ideal gear pair can be modeled, as shown in Figure $2 \mathrm{a}$, by a rigid cam having a displacement $R_{b 1} \Delta \theta$ and moving horizontally over rigid springs. The springs represent the tooth pairs and are therefore spaced by one normal pitch $p_{n}$. A spring coming in contact with the cam profile represents the formation of a tooth pairs while a spring leaving contact with the cam profile represents the parting of a tooth pair. The straight part of the cam AB represents the line of action of the gear pair. The cam profile outside the straight portion AB represents the separation distance $\Delta S$ between teeth and it describes the manner in which teeth approach and recede from each other.
As the contact starts at the bottom of tooth $a_{1} a_{2}$ at the start of the meshing cycle (position (1)), the separation distance $\Delta S d$ of the tooth pair $d_{1} d_{2}$ is too small for this pair of teeth to enter in contact prematurely, at position (2), under the applied torque. This contact lasts until an $S$ value of $1.38 p_{n}$ is reached, and for which $\Delta S_{d}$ of tooth pair $d_{1} d_{2}$ becomes greater than the cam vertical displacement, $R_{b 1} \Delta \theta$, at position (4). At this instant of time, the gears have two pairs of teeth in contact, lasting until $S / p_{n}$ becomes equal to $1.40 p_{n}$, for which $R_{b 1} \Delta \theta$ becomes greater than $\Delta S_{c}$ of tooth pair $c_{1} c_{2}$. The latter enters in premature contact at position (5). The pair of teeth, $c_{1} c_{2}$ come into full contact at position (1), and the cycle is repeated. Figure $2 \mathrm{~b}$ shows positions (1), (3) and (5) of the gears, as well as the intermediate position, between positions (3) and (4), where only two pairs of teeth are in contact. Figure $2 \mathrm{~b}$ also shows that $c_{1} c_{2}$, at position (5), enter in contact prematurely.

Subsequently, we can determine the real contact ratio by considering (see Fig. 2), that the real point of the beginning and at the end of contact will be point $A^{\prime}$ (with the highest $S_{1 \max }$ measured on the line of action to the left of pitch point $P$ ) and $B^{\prime}$ (with the highest $S_{2 \max }$ on the line of action to the right of $P$ ), rather than points $A$ and $B$ which are the theoretical points at the beginning and the end of contact, respectively. The real contact ratio will then be

$$
\varepsilon_{r}=\frac{S_{1 \max }+S_{2 \max }}{p_{b}}
$$




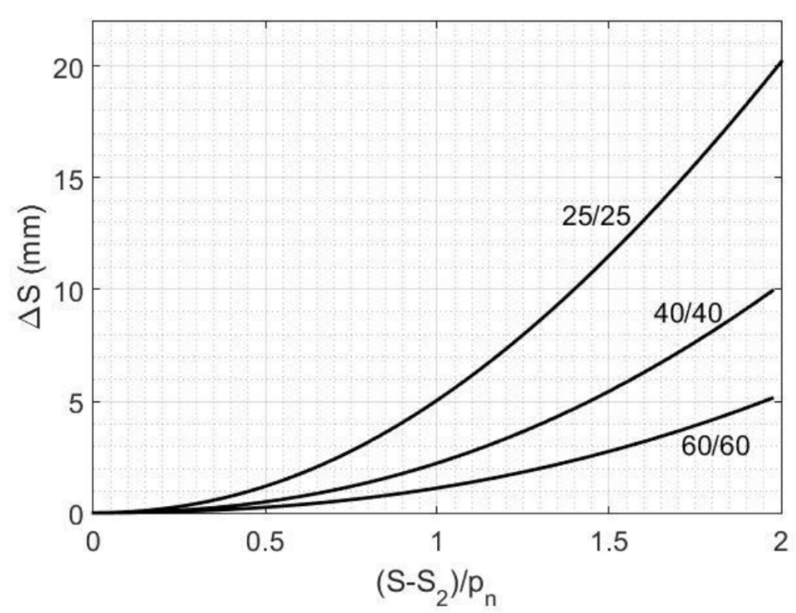

(a)

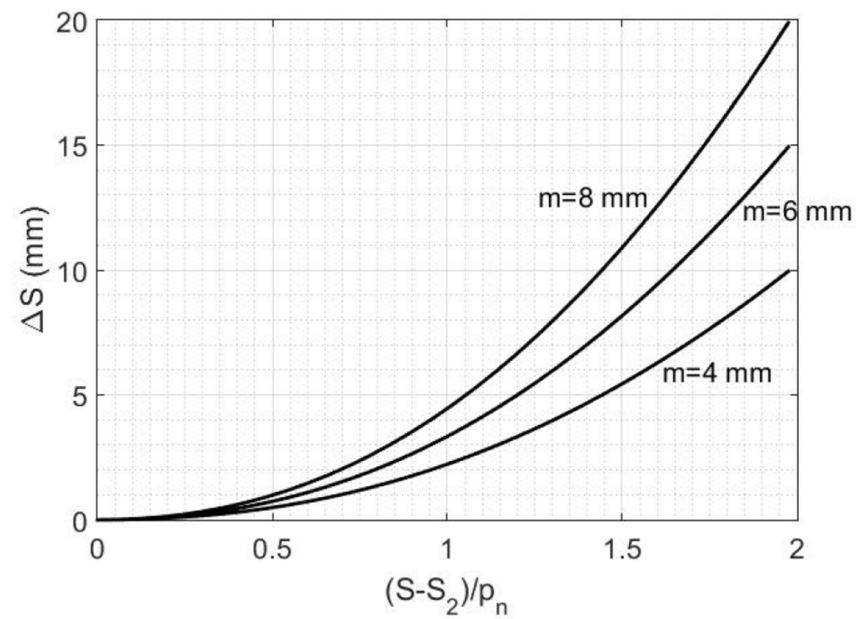

(b)

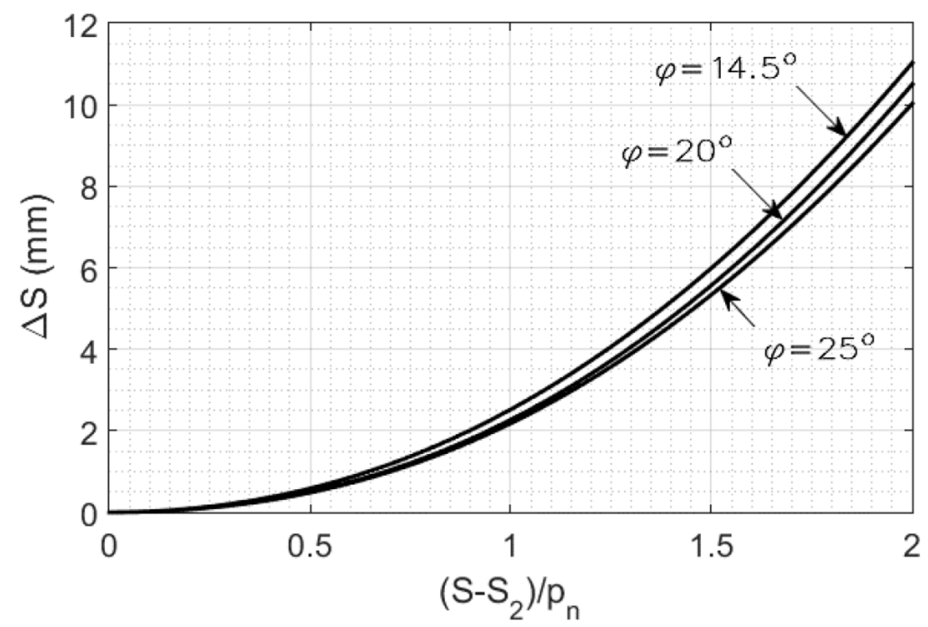

(c)

Fig. 3. Effects of the gear parameters on the variation of the separation distance $\Delta S$.

The real contact ratio in this case is 2.94 , compared to the ideal contact ratio of 1.78 given by the abscissa length $\mathrm{AB}$.

Equation (6) can also be obtained by replacing $p_{b}$ by $\pi m \cos \varphi$ and $\mathrm{S}$ by $R_{b 1} \psi$ (Eq. (9) in the appendix), where $\psi$ stands for the tooth position relative to the center-to-center line of the gear pair, as shown in Figure 20 in the appendix, and $R_{b 1}=N_{1} m \cos \varphi / 2$.

This enables one to write

$$
\varepsilon_{r}=\frac{\left|\psi_{1 \max }\right|+\left|\psi_{2 \max }\right|}{\frac{2 \pi}{N_{1}}}
$$

The effective contact ratio is then defined, as shown in equation $\left(6^{\prime}\right)$, as a ratio of the rotation angle between the first and last tooth contact points to the pinion angular pitch. The accuracy of the proposed method for calculating real contact ratio depends on the method used to calculate separation distance $\Delta S$ and the compliance w of a tooth pair. First consider the calculation of separation distance.

\section{Method of calculation of the separation distance $\Delta S$}

The proposed method, in this paper, for the calculation of the separation distance between teeth which are not initially in contact, can also be used to calculate the radii of contact between the pinion and the gear after the pinion has rotated an angle $\Delta \theta$. For this to happen, two cases are considered:

- The case where the head of the gear tooth enters in contact prematurely with the root of the pinion tooth. This is represented by $q_{1} q_{2}$ in Figure 1 .

- The case where head of the pinion enters in contact with the root of the gear tooth. This is represented by $k_{1} k_{2}$ in Figure 1.

The suggested method to calculate the separation distance is shown in the appendix. This separation distance varies as a function of the module, the number of teeth and the pressure angle, as depicted in Figure $3 \mathrm{a}-\mathrm{c}$. Figure 3-a shows the variation of the separation distance for $25 / 25$, 


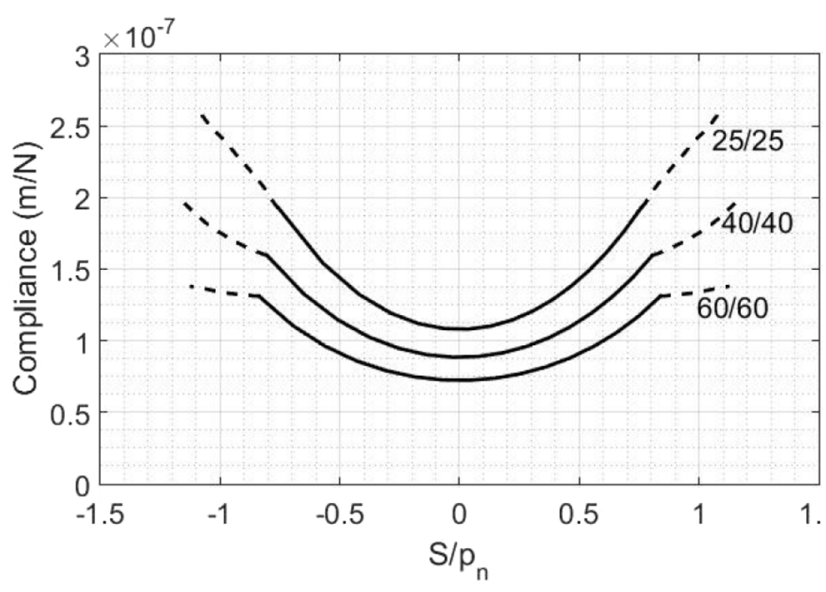

(a)

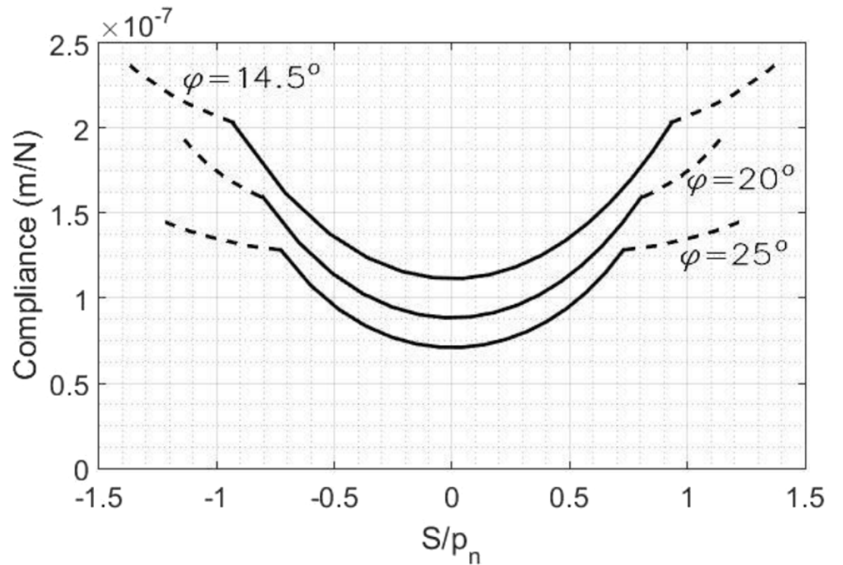

(b)

Fig. 4. Effects of increasing the number of teeth and the pressure angle on the compliance of plastic gear pairs.

$40 / 40$ and $60 / 60$ gears, all with a $5-\mathrm{mm}$ module and a $20^{\circ}$ pressure angle. Figure $3 \mathrm{~b}$ also shows the variation of $\Delta S$ for module values of 4,6 and $8 \mathrm{~mm}$, for a $40 / 40$ pair of gears and a pressure angle of $20^{\circ}$. Figure $3 \mathrm{c}$ shows the variation of $\Delta S$ with pressure angle values of $14.5^{\circ}, 20^{\circ}$ and $25^{\circ}$ for a $40 /$ 40 gear pair and module of $4 \mathrm{~mm}$. The abscissa in these figures is the distance $\left(S-S_{2}\right) / p_{n}$ where $S$ is the distance, along the line of action, from the contact point to the primitive point $P$ (see Fig. 20 in the appendix), and $S_{2}$ is the distance from the theoretical contact point located to the right of point $P$ (point $\mathrm{B}$ in Fig. 2).

\section{Compliance of a tooth pair}

In ISO standard 6336 [1,2] as well as in other works [14-17], only a simplified model of the tooth is considered for the calculation of the deflection of the tooth profile. This model of tooth represents a trapezoidal rack tooth with the same standard basic rack tooth profile as the gear. In other works $[18,19]$, the potential energy method is employed to evaluate the meshing stiffness while in some works $[5,20]$ finite element analysis is used for the tooth stiffness estimation. The adopted tooth model, in this paper, is based on the work of Yelle and Burns [4] which, itself, is based of the results found by Timoshenko and Baud [17] and further verified experimentally by Furrow and Mabie [21].

The compliance, at each position of the point of contact on the pair of meshing teeth, can be expressed as:

$$
w=w_{t 1}+w_{t 2}
$$

where

$$
w_{t 1}=w_{b 1}+w_{s 1}+w_{a 1}
$$

and

$$
w_{t 2}=w_{b 2}+w_{s 2}+w_{a 2}
$$

$w_{t 1}$ and $w_{t 2}$ are the tooth compliance of the driving and driven gears, which includes the bend compliance $w_{b}$, the shear compliance $w_{s}$ and the axial compressive compliance $w_{a}$, where subscripts 1 and 2 denote the driving and driven gears. More detailed descriptions for $w_{b}, w_{s}$ and $w_{a}$ considering can be found in reference [4].

The compliances of a pair of contacting teeth vary as a function of the position of this pair along the line of action. Figure $4 \mathrm{a}$, shows the variation of the compliance by increasing the number of the teeth of the pinion and the gear. We have here a $4-\mathrm{mm}$ module, a $20^{\circ}$ pressure angle and a face width of $30 \mathrm{~mm}$, for a Nylon 66 material with a modulus of elasticity of $3.08 \mathrm{MPa}$ [22]. The abscissa in this plot is the normalized value $S / p_{n}$. Figure 4-a also shows, in dashed line, the compliance of the teeth which could enter in contact prematurely to the left and right of the primitive point $P$, at values of $S / p_{n}>0.8$ or $S / p_{n}<-0.8$. Figure $4 \mathrm{~b}$ shows the variation of the compliance (for Nylon 66) by varying the pressure angle, taking the module to be $4 \mathrm{~mm}$, considering a 40/40 gear ratio and assuming a face width of $30 \mathrm{~mm}$. Since the gears are identical, the tooth pair compliance $w$ curve is symmetrical about $S / p_{n}=0$. One can see from these figures that the compliance decreases when the number of pinion and gear teeth increases and when the pressure angle increases.

We show also in Figure 5 the variation of the compliance for a case where the pinion is made of plastic and the gear is made of steel. Here, the module is $4 \mathrm{~mm}$, the pressure angle is $20^{\circ}$, the face width is $30 \mathrm{~mm}$ and the gear ratio is $20 / 30$. One notices that the much larger modulus of steel makes it reasonable to assume that it is perfectly rigid, i.e. $w_{2} \sim 0$, and the total compliance curve represents that of a plastic tooth.

\section{Finite element modeling and calculation}

In addition to the analytical method, the finite element method is used for the calculation of the real contact ratio and the tooth root stress, caused by the applied torque. This allows load-induced displacements to be taken into 


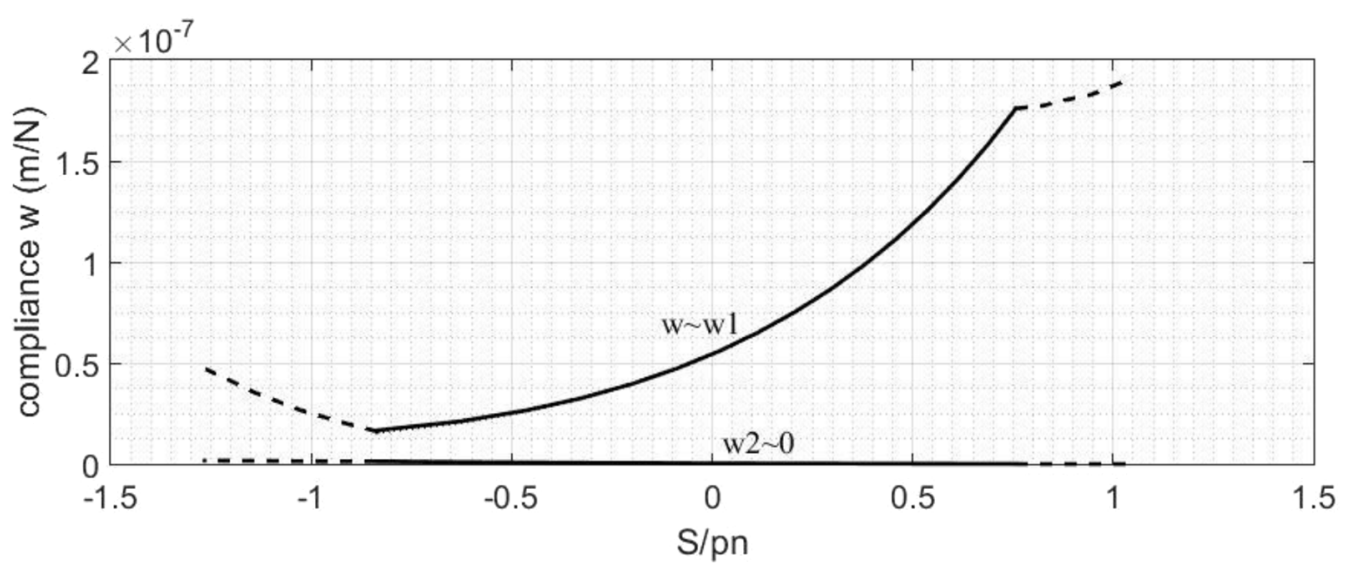

Fig. 5. Variation of the compliance for the $20 / 30$ plastic/steel gear pair.

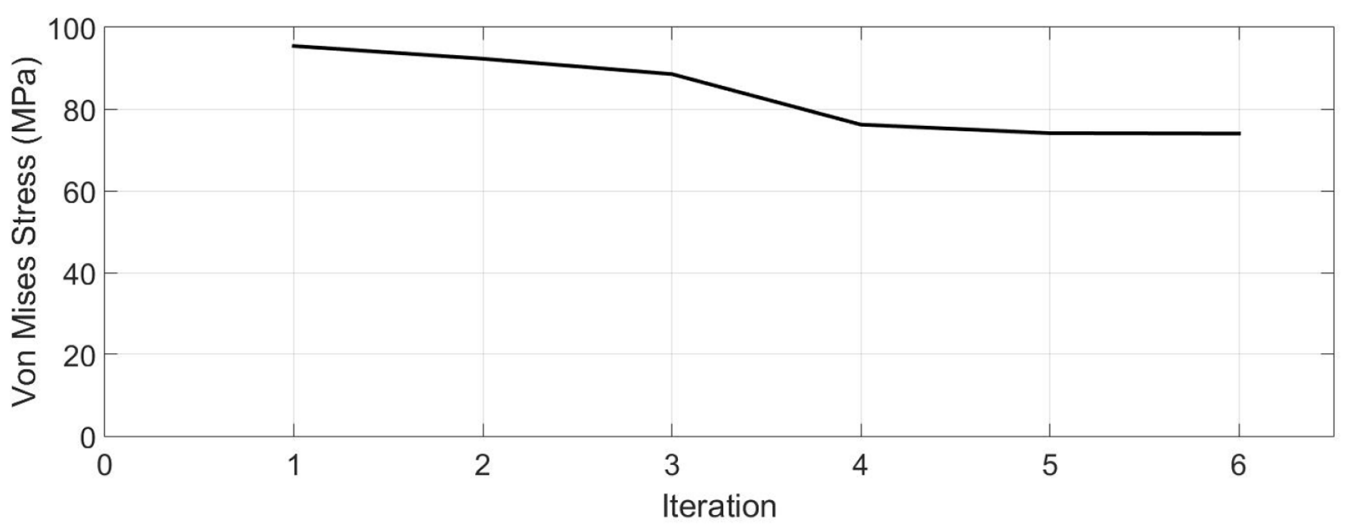

Fig. 6. Convergence of the values of the Von Mises stress for the $40 / 40$ pair of gears.

account, assuming the appropriate boundary conditions are chosen. The method of generation of the finite element model can be described as follows:

- The geometry of each of the two gears, as well as their assembly, is created using Solidworks ${ }^{\circledR}$. A joint gear is associated to enable the simultaneous rotation of the two gears with consistent gear ratio and direction of rotation. This allows performing finite element calculation in accordance with the kinematics of the pair of gears in contact.

- The simulation software integrated in SolidWorks ${ }^{\circledR}$ is used to create the mesh and to perform the stress analysis. It has also the capability of simulating large displacements in the elastic range. The FEM results, thus obtained, are used to validate those obtained from the analytical model.

The finite element solution has been obtained via Solidworks which meshes the gears using 10-noded tetrahedral elements (one node at each of the four corners and six mid-side nodes). The element possesses three DOF at each node. This 3-dimensional model should adequately represent the behavior of the meshed gears, particularly, as regards bending of the tooth. The program, also, enables one to manually track, through an internal algorithm, called "Trend Tracker", the variation of the Von Mises stress for each iteration. Afterwards, if the stress does not converge to the desired value, the mesh is refined, particularly, at locations of high stress gradients near the fillet and at the gear contact area, and a new iteration is started. The convergence of the solution is deemed satisfactory when a flat curve in a Von Mises stress vs. number of iterations plot is obtained. It can be seen from Figure 6, for the 40/40 pair of gears, that the curve almost flattens (at a stress $=75 \mathrm{MPa}$ ) following the sixth iteration resulting in an element size of $0.2 \mathrm{~mm}$, keeping a constant aspect ratio of 1 . It should also be mentioned that 177,785 tetrahedral elements are used to mesh the gears at the sixth iteration.

Figure 7 shows an example of the finite element model of the $40 / 40$ pair of gears in contact obtained by this method for a particular position of the teeth during a meshing cycle.

\section{Results}

A computer program using MATLAB is developed to assess the validity of the proposed method. In this study, the NYLON 66 is chosen as a material of reference. This material has a tensile yield strength of $120 \mathrm{MPa}$ [22]. On the other hand, a parametric investigation is carried out to 


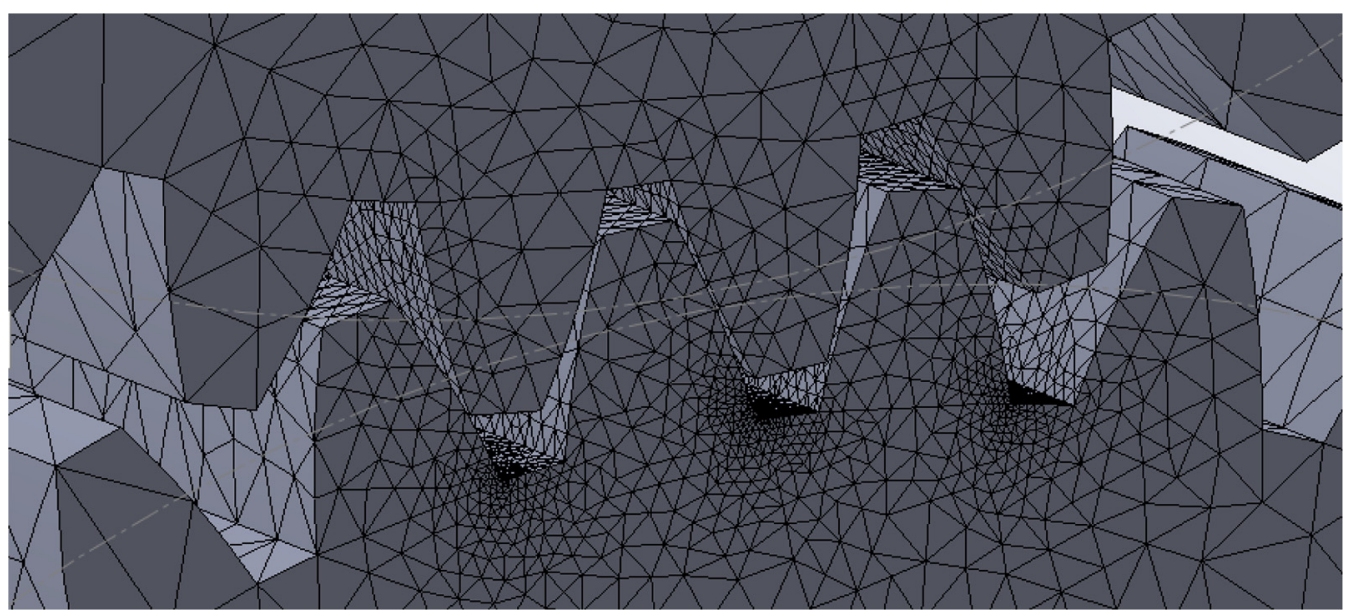

Fig. 7. Regions of local meshing of a $40 / 40$ pair of gears.

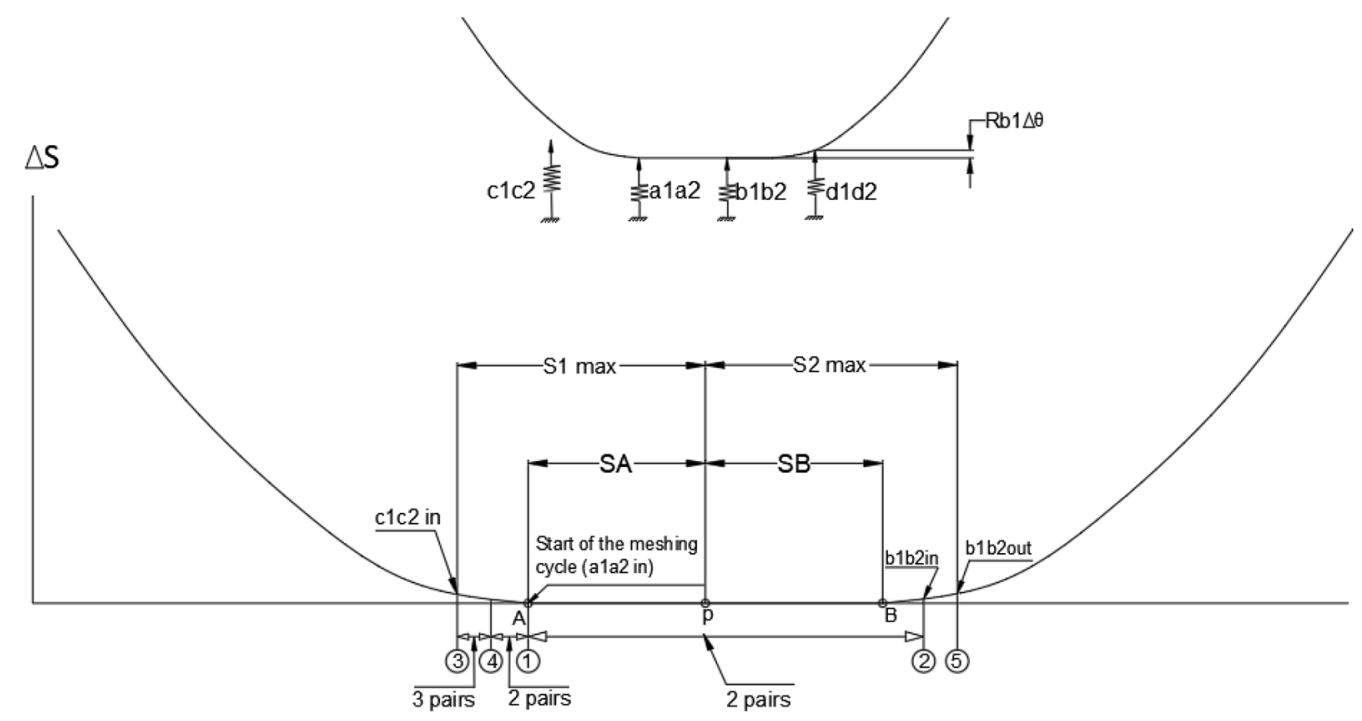

Fig. 8. Behavior of a Nylon 66 pair of plastic gears with 25 teeth each with an applied.

study the effect of increasing the number of teeth of both the pinion and the gear, of the module and of the pressure angle on the variation of the real contact ratio and on the variation of the bending stress.

\subsection{Real contact ratio}

\subsubsection{Validation of the proposed method}

In Section 3, we discussed the proposed method for analyzing the real behavior of a pair of meshed plastic gears. The method yielded results which were verified by finite element calculations.

Figure 8 depicts the real behavior of a pair of $25 / 25$ gears, obtained by the proposed method, under a torque of $300 \mathrm{~N} \mathrm{~m}$. The module and the face width are equal to $4 \mathrm{~mm}$ and $30 \mathrm{~mm}$, respectively. For a meshing cycle which begins at the outset of contact between teeth $a_{1} a_{2}$, equation (4) shows that, between positions (1) and (2), the vertical displacement of the cam is less than the separation distances $\Delta S_{c}$ and $\Delta S_{d}$ between the tooth pairs $c_{1} c_{2}$ and $d_{1} d_{2}$. Consequently, there are two pairs of teeth in contact $\left(a_{1} a_{2}\right.$ and $\left.b_{1} b_{2}\right)$ between these positions. When the tooth pair $c_{1} c_{2}$ reach position (3), the value of $R_{b 1} \Delta \theta$ becomes greater than the separation distance $\Delta S_{c}$ between this tooth pair which enter in contact prematurely. At position (5), the tooth pair $b_{1} b_{2}$ completely disengage while the tooth pair $c_{1} c_{2}$ reach position (4) and, subsequently, come into full contact at position (1), and the cycle is repeated. Figure 8 shows that between positions (3) and (4), there are three tooth pairs in contact. However, between positions (1) to (2), and (4) to (1), two pairs are in contact. At position (3), $S_{1 \max }$ is $18.4 \mathrm{~mm}$, whereas, $S_{2 \max }$ is $16.9 \mathrm{~mm}$ at position (5). The real contact ratio, calculated from equation (6), is 2.34 compared to 1.71 for the ideal gear pair.

The same results obtained by FEM can be seen in Figure 9 for the 5 aforementioned positions. This figure shows positions (1), (3) and (5), as well as the intermediate position, between positions (4) and (1), where only two pairs 

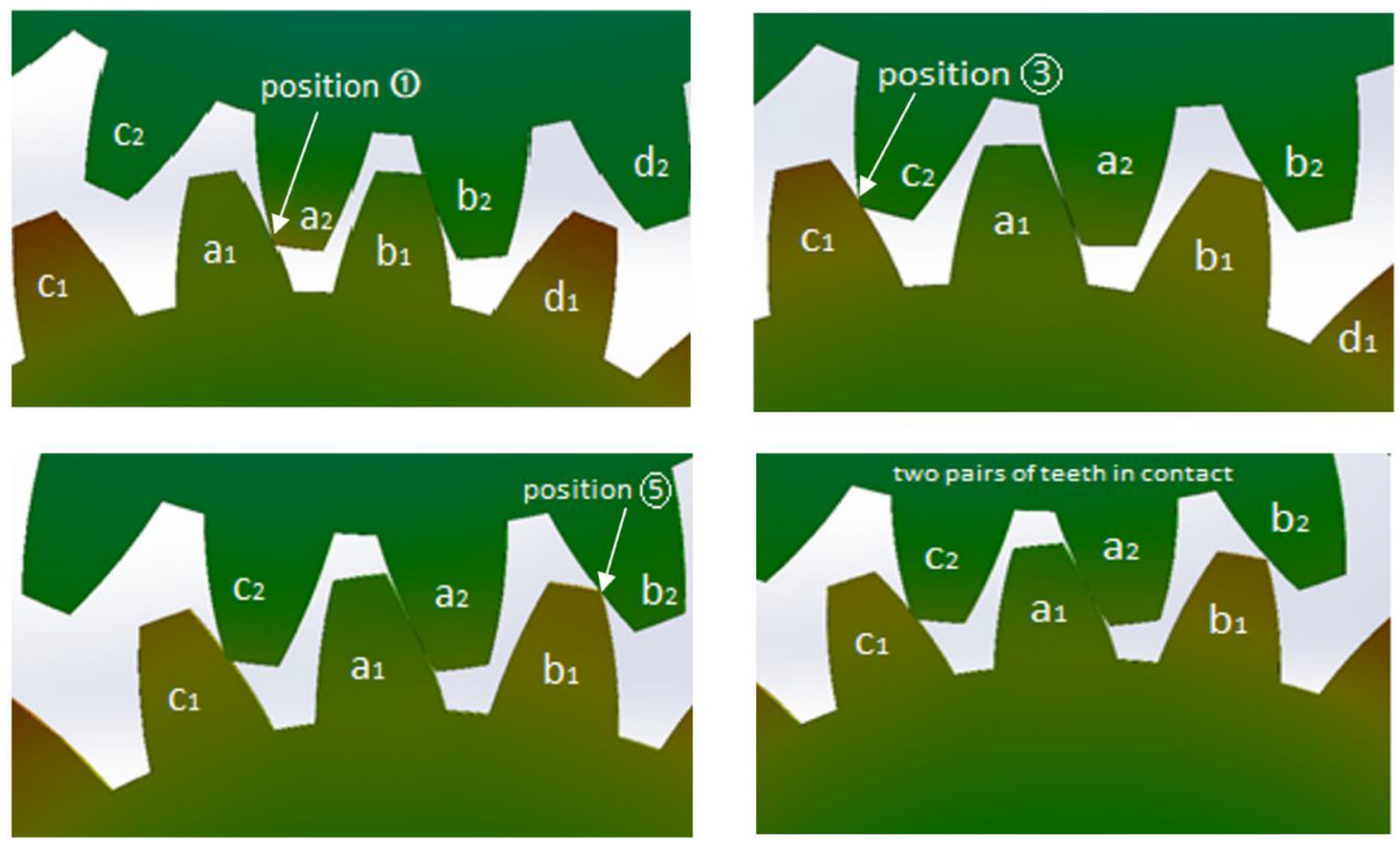

Fig. 9. Results obtained by the FEM for the pair of 25/25 plastic gear pair.
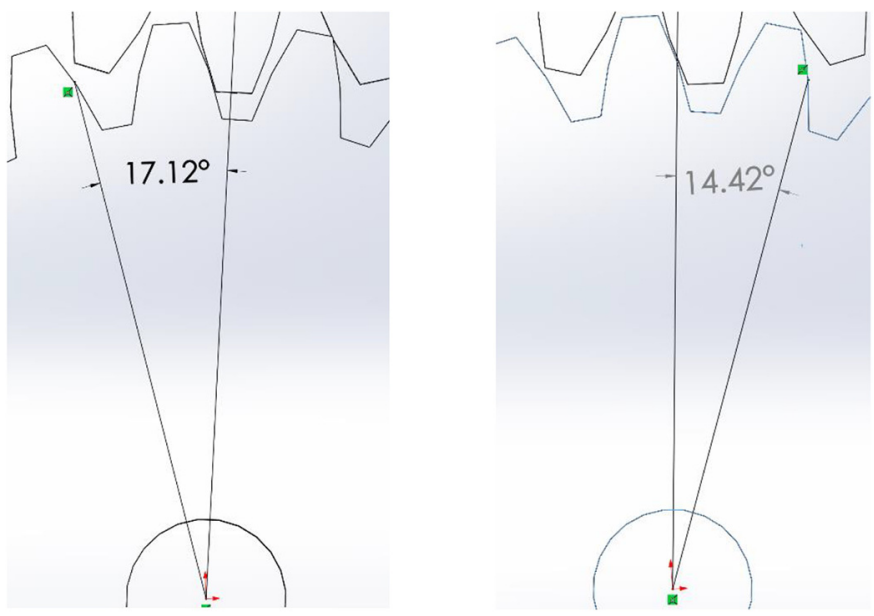

Fig. 10. Values of $\psi_{1 \max }$ and $\psi_{2 \max }$ obtained by FE of the $25 / 25$ pair of plastic gears.

of teeth are in contact. Figure 9 also shows that $c_{1} c_{2}$, at position (3), enters in contact prematurely.

The value of $\psi_{1 \max }$, pertaining to $c_{1} c_{2}$ (position (3) in Fig. 10), is $17.12^{\circ}$ whereas the value $\psi_{2 \max }$, pertaining to $\mathrm{b}_{1} \mathrm{~b}_{2}$ (position (5) in Fig. 10) is $14.42^{\circ}$. The aforementioned values correspond to a real contact ratio of 2.18 , obtained from equation $\left(6^{\prime}\right)$. This ratio is close to the one calculated using the analytical method, which validates the proposed method.

Figure 11 shows the effect of changing the gear ratio from $25 / 25$ to $40 / 40$ under a torque of $500 \mathrm{Nm}$. A comparison of Figures 8 and 11 reveals that the two pairs of gears behave differently. At the start of the meshing cycle, when the pairs of teeth $a_{1} a_{2}$ and $d_{1} d_{2}$ are at positions (1) and (2), respectively, $R_{b 1} \Delta \theta$ is such that the cam's profile makes contact with $d_{1} d_{2}$ at position (2). The pair of Teeth $d_{1} d_{2}$ remain in contact until reaching position (3), at which point, the deflection of pairs $\mathrm{a}_{1} \mathrm{a}_{2}$ and $b_{1} b_{2}$ becomes less than the separation distance $\Delta S_{\mathrm{d}}$ of tooth pair $d_{1} d_{2}$ which ceases contact at this position. When the pair $c_{1} c_{2}$ reach position (5), $\Delta S_{\mathrm{c}}$ becomes less than the vertical displacement of the cam. Consequently, this pair of teeth enter in contact prematurely. This contact lasts until reaching position (1). The cycle is then repeated. Figure 11 also shows that, there are three pairs of teeth in contact between positions (1) to (3), and (5) to (1). This is despite the fact that, at position (2), the pair of teeth $\mathrm{d}_{1} \mathrm{~d}_{2}$ has overshot point $\mathrm{B}$ located at the end of the line of action. In addition, Figure 11 shows that only two pairs of teeth remain in contact between positions (3) and (4). The real contact ratio, calculated from equation (6), is 2.96 compared to 1.78 for the ideal gear pair.

The same results obtained by FEM can be seen in Figure 12, for the 5 aforementioned positions. The real contact ratio, calculated from equation $\left(6^{\prime}\right)$, is 2.87 .

Figure 13 shows the effect of keeping the gear ratio at $40 / 40$ but using steel for the driven gear. The much larger modulus of steel makes it reasonable to assume that is perfectly rigid, i.e., $w_{2}=0$, and the total compliance curve reduces to that of the plastic tooth. Figure 13 also shows the behavior of this plastic/steel gear pair. In this case the real contact ratio is 2.47 compared to 2.96 for the equivalent plastic/plastic gear pair. For this plastic/steel combination and load, one still has as many as 3 tooth pairs in contact but the duration of such contact is less than that experienced by the equivalent plastic/plastic gear pair. 


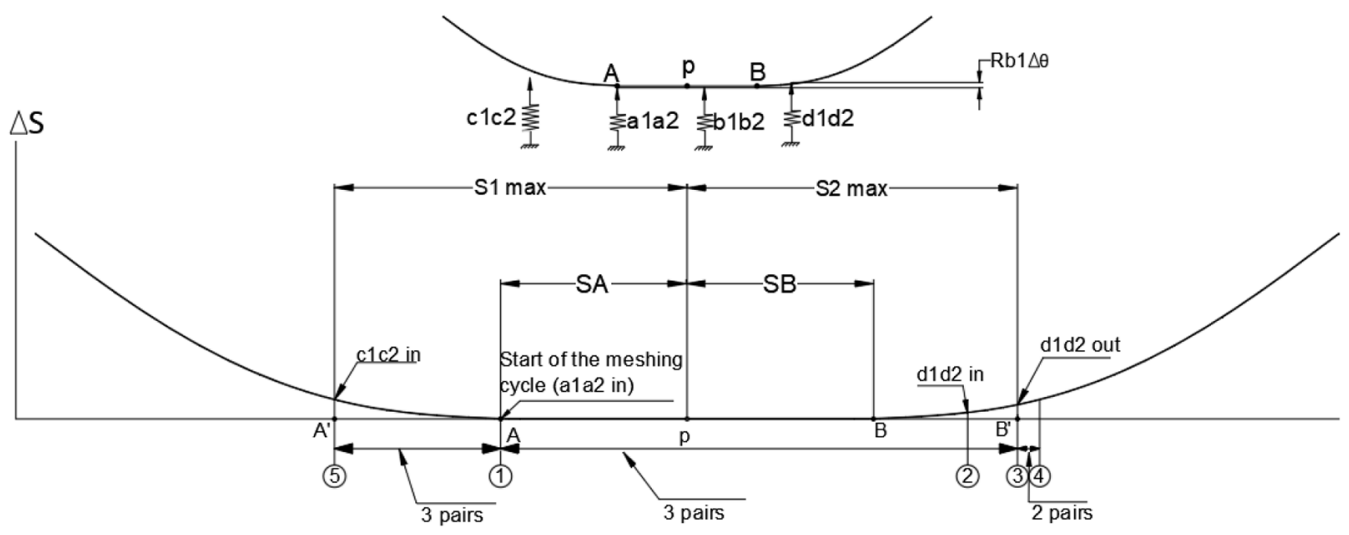

Fig. 11. Behavior of a Nylon 66 pair of plastic gears with 40 teeth each with an applied torque of $500 \mathrm{Nm}$.
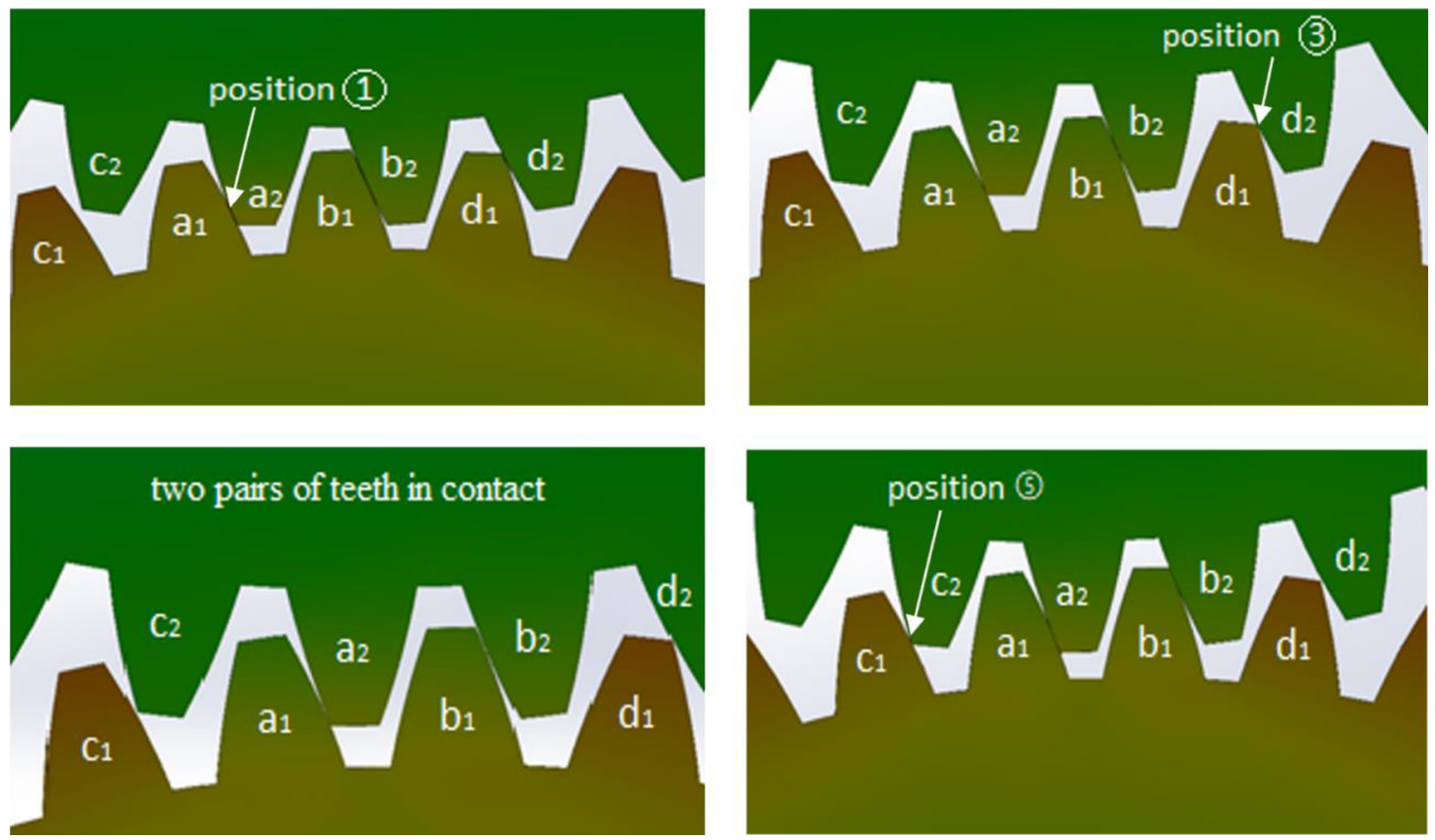

Fig. 12. Results obtained by the FEM for the pair of $40 / 40$ plastic gear pair.

\subsubsection{Effect of gear parameters on the contact ratio}

From Figure 2 and Figures 8 through 13, it is clear that the real contact ratio varies depending on the number of teeth of the meshing gears and on the applied torque. Similarly, the change of module, and of the pressure angle also have the effect of changing the value of the real contact ratio. For this reason, the effect of number of teeth, module, and pressure angle variation are discussed in the following where different types of gears are considered whose characteristics were mentioned in Sections 4 and 5 .

Figure 14a shows the variation of the real contact ratio obtained as a function of the applied torque for $25 / 25$, $40 / 40$ and $60 / 60$ gears, all with a $4-\mathrm{mm}$ module and a $20^{\circ}$ pressure angle. In this figure, it can be seen that the real contact ratio increases with the increase in the number of teeth of the pinion and of the gear. This increase can be explained by the fact that the increase in the number of teeth of gear will cause the increase of its outer radius and the decrease of the separation distance $\Delta S$ as shown in Figure 3a. This increase of the outer radius and this decrease of $\Delta S$ will compensate for the decrease in the torsion angle $\Delta \theta$ due to the increase in stiffness with the increase in the number of teeth as shown in Figure 4a.

Figure $14 \mathrm{~d}$ shows the variation of the real contact ratio in the case where the pinion is made of plastic and the gear is made of steel (with a Young's modulus of $210 \mathrm{GPa}$ ). It can be seen from this figure that the same behavior exists in the case of plastic/plastic gears, but the contact ratio is lower. This result may be justified by the fact that the increase in the stiffness of the teeth of the gear is due to the increase in its modulus of elasticity which has the effect of reducing the number of teeth that come into contact prematurely. 


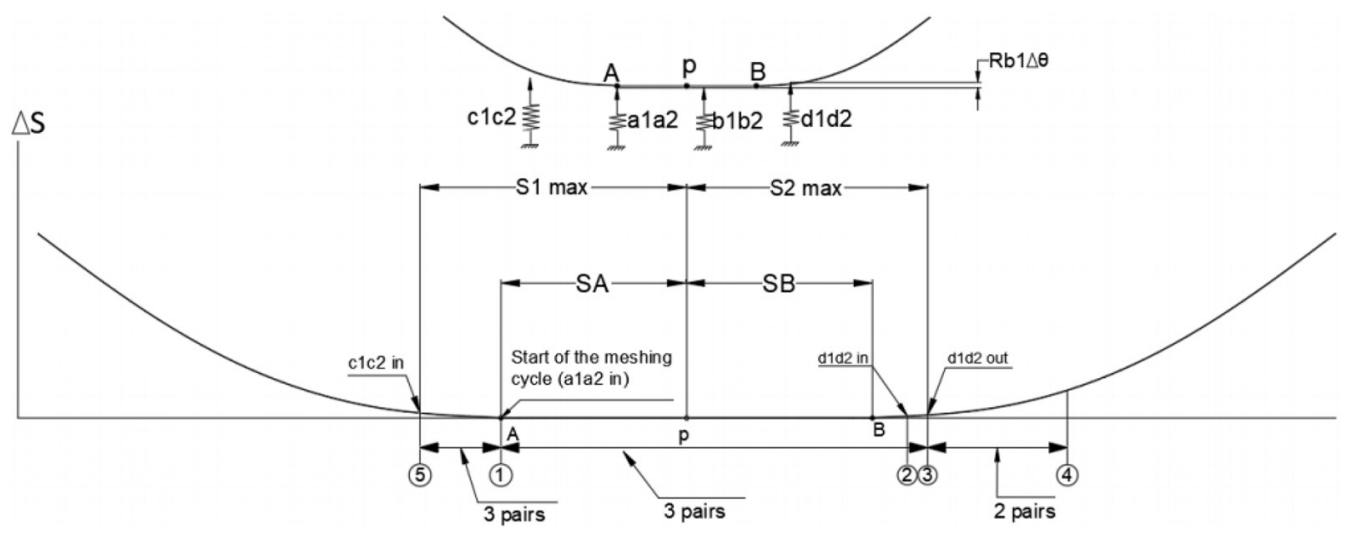

Fig. 13. Behavior the $40 / 40$ plastic/steel gear gear for an applied torque of $500 \mathrm{Nm}$.

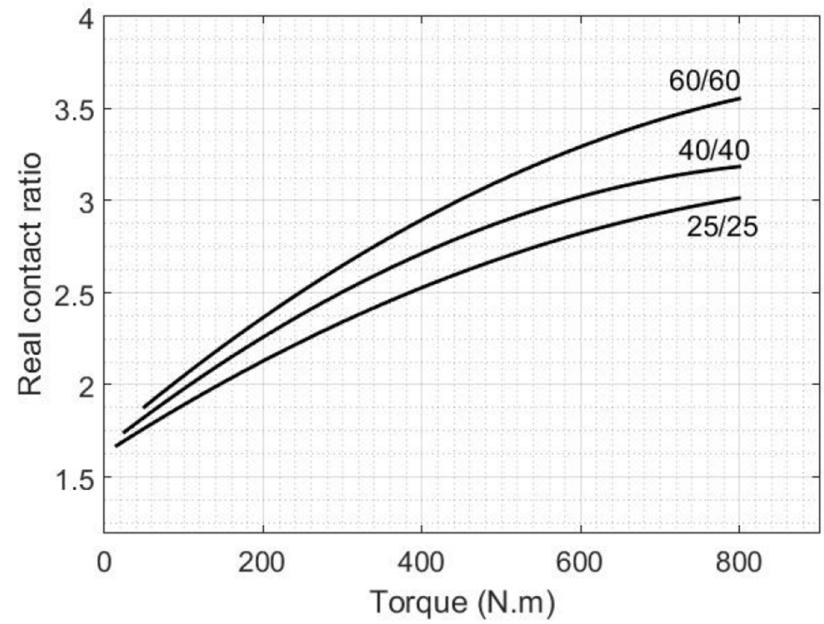

(a)

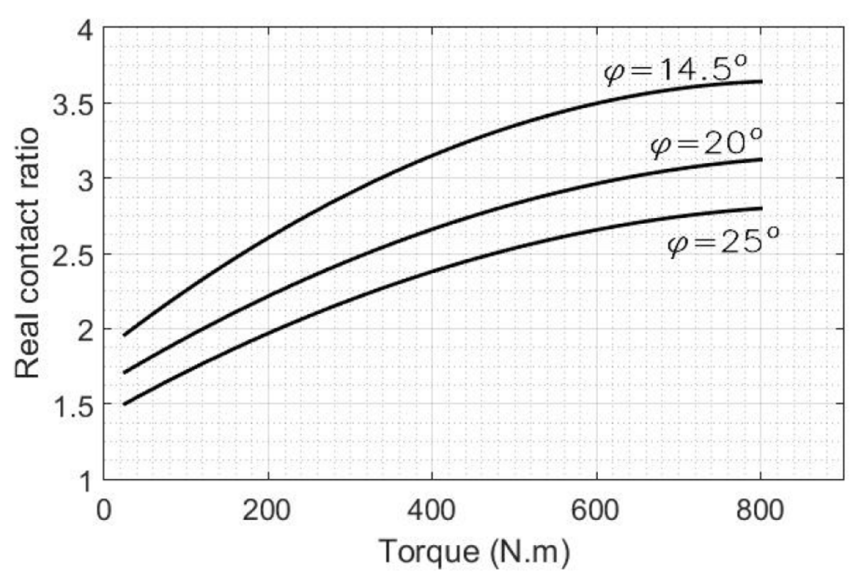

(c)

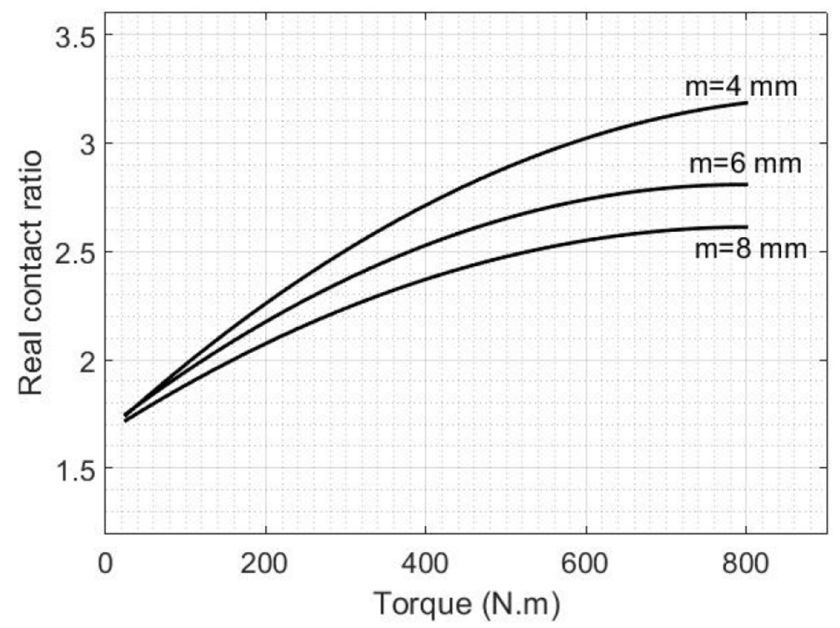

(b)

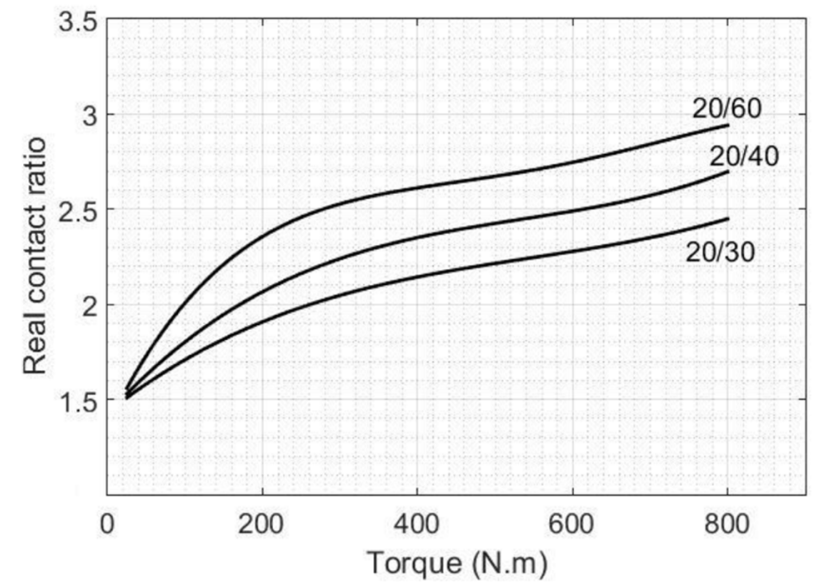

(d)

Fig. 14. Effect of gear parameters on the variation of the real contact ratio as a function of the applied torque.

As for the effect of the variation of the module $m$ on the behavior of the plastic spur gears, three values of the module of $4 \mathrm{~mm}, 6 \mathrm{~mm}$ and $8 \mathrm{~mm}$ are considered and the values of the real contact ratio is determined as a function of the applied torque. The results obtained are shown in Figure 14b. It can be noticed, in this figure, that the real contact ratio increases with an increase of the module. This can be explained by the decrease of the separation distance $\Delta S$ as shown in Figure 3c.

The effect of the variation of the pressure angle $\varphi$, on the behavior of plastic spur gears is also studied. Figure $14 \mathrm{c}$ shows the variation of the real contact ratio, for a $40 / 40$ 


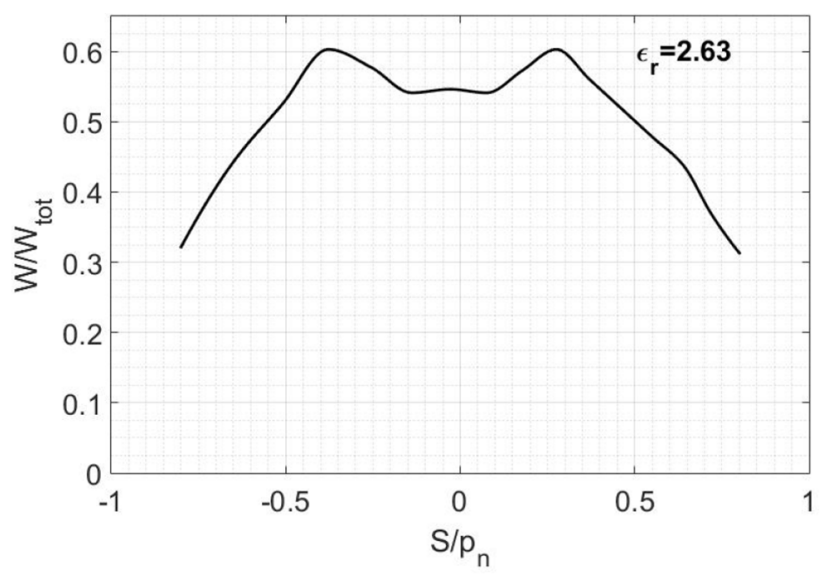

(a)

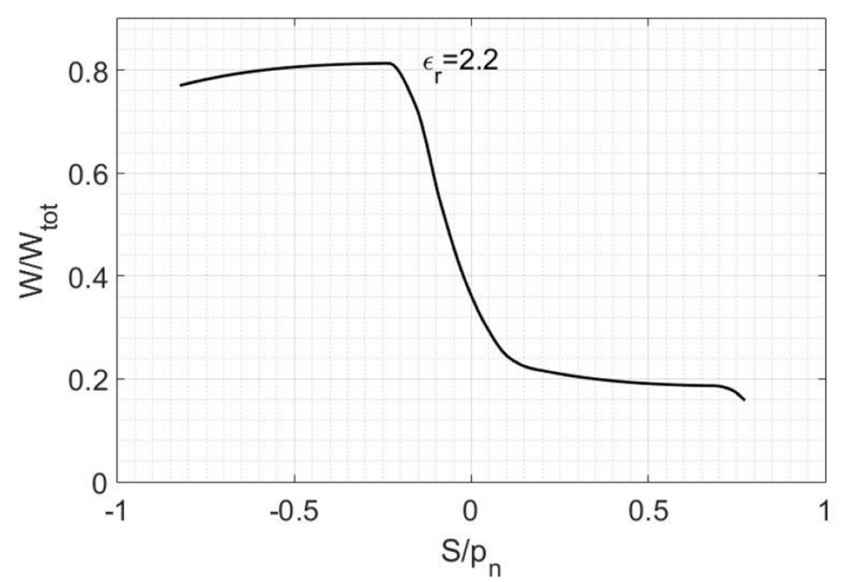

(b)

Fig. 15. Load sharing for the $25 / 25$ plastic gear pair and the $20 / 30$ plastic/steel gear pair for different contact ratios.

pair of plastic gears, as a function of the applied torque, for values of the pressure angle $\varphi$ of $14.5^{\circ}, 20^{\circ}$ and $25^{\circ}$. It can be seen, from this figure, that the real contact ratio decreases with increasing pressure angle. The decrease of the real contact ratio can be related to the rate of increase of the stiffness of the tooth which is greater than the rate of decrease of the separation distance $\Delta S$ as shown in Figures $3 \mathrm{~b}$ and $4 \mathrm{~b}$, which represent the variation of $\mathrm{w}$ and the variation of $\Delta S$ for the three values of $\varphi$, mentioned above.

It should be noted that the direct effect of the increase in the real contact ratio is to reduce the loads on the teeth that are initially in contact. The load on a line which is initially in contact can be calculated from equation (1) after calculating the distortion angle $\Delta \theta$ and the compliance $w$. For this reason, during a meshing cycle on a tooth where the contact begins at the lower point on the latter and ends at its tip, it is possible to determine the variation of the load by calculating the various values of $\Delta \theta$ by using equation (4).

Figure 15a shows the variation of the load on a contact line between the beginning and the end of contact in the case where the pinion and the gear are made of plastic. The two gears are $25 / 25$, they have a modulus of $4 \mathrm{~mm}$ and a pressure angle of $20^{\circ}$. The abscissa of this load plot is normalized as $S / p_{n}$. This figure shows the variation of the ratio of the load $W$ on the total load transmitted by the applied torque which is defined by $W_{t o t}=T / R_{b 1}$. It may be noted, from this figure, that for a torque of $500 \mathrm{~N} \mathrm{~m}$, which corresponds to a real contact ratio of 2.56, according to Figure $15 \mathrm{a}$, a line of contact supports at most about $60 \%$ of the total normal load.

Figure 15b also shows the variation of the load on a line of contact in the case where the pinion is made of plastic and the wheel is made of steel. The two gears have a modulus of $4 \mathrm{~mm}$, a pressure angle of $20^{\circ}$ and a ratio of number of teeth of $20 / 30$. we note, here, that for a torque of 400 N.m, corresponding to a real contact ratio of 2.2 (see Fig. 14d), no teeth pair carries more than $80 \%$ of the total normal load. This value is obtained near the area where the value of the compliance $\mathrm{w}$ is minimal (see Figs. 5 and 15b).

\subsection{Bending stress}

\subsubsection{Case of plastic/plastic spur gears}

The calculation procedure proposed in this article makes it possible to calculate the bending stress at each position of the point of contact. This bending stress is obtained from the equation used for a trapezoidal beam $[1,4]$

$$
\sigma_{F}=\frac{6 W_{t r} \cos \alpha_{F} h_{F}}{F S_{F}^{2}}
$$

where $W_{t r}$ is the transverse load, $\alpha_{F}$ is the load angle, $h_{F}$ is the bending moment arm, $\mathrm{F}$ is the face width, and $S_{F}$ is the thickness of the tooth at the critical section (whose tangent to the root fillet forms an angle of $30^{\circ}$ with the tooth centerline).

For the same characteristics of the gears in the sections 4 and 5 and for a torque of 500 N.m, Figure 16a through c shows the variations of the bending stress on a line of contact during a meshing cycle relative to the rotation of the gear between the beginning and the end of contact on this line. The plots in these figures are generated from both the theoretical and the finite element model. Figure 16a shows the effect of increasing the number of teeth of the pinion and of the gear. Figure $16 \mathrm{~b}$ shows the effect of increasing the module, and Figure 16c shows the effect of increasing the pressure angle. The general trend in these figures is the same, although the bending stress values are different; they are smaller for a larger module, larger number of teeth and larger pressure angle. Note the very good agreement obtained between the analytical and finite element models.

From Figure $16 \mathrm{a}-\mathrm{c}$, we can see that the maximum bending stress is located near the point of contact at the middle of the path of the line of contact between the primitive point $P(S=0)$ and the end of contact on the outer radius of the pinion (point B in Fig. 2 located at the distance $S_{2}$ to the right of the point $P$ ). Very similar results were obtained from a study with several combinations of gear parameters which do not necessarily have the same number of teeth with the observation that the 


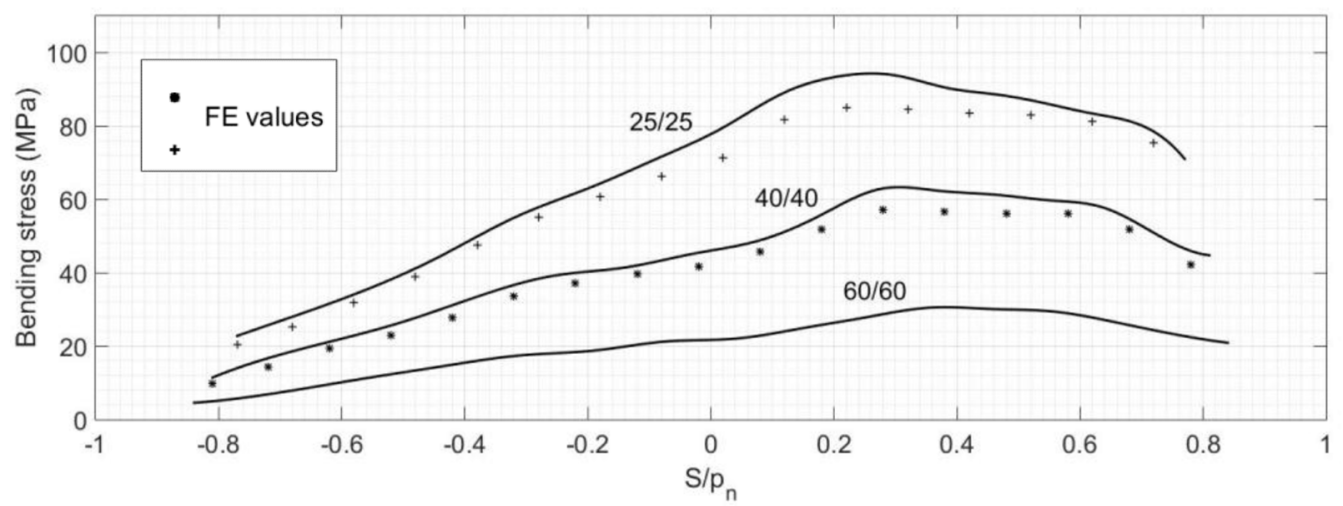

(a)

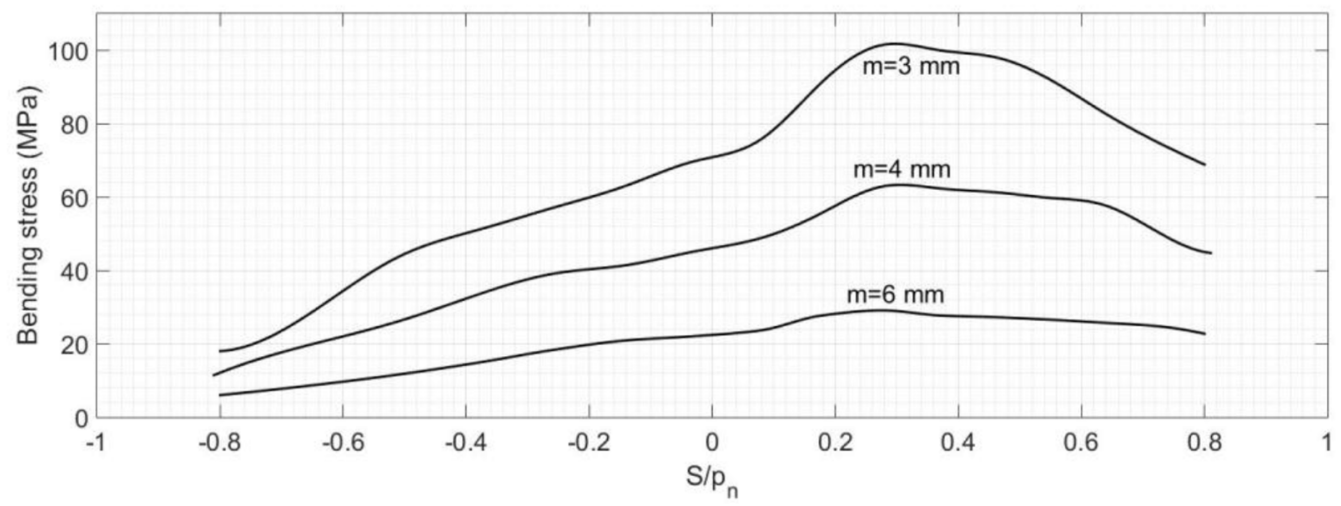

(b)

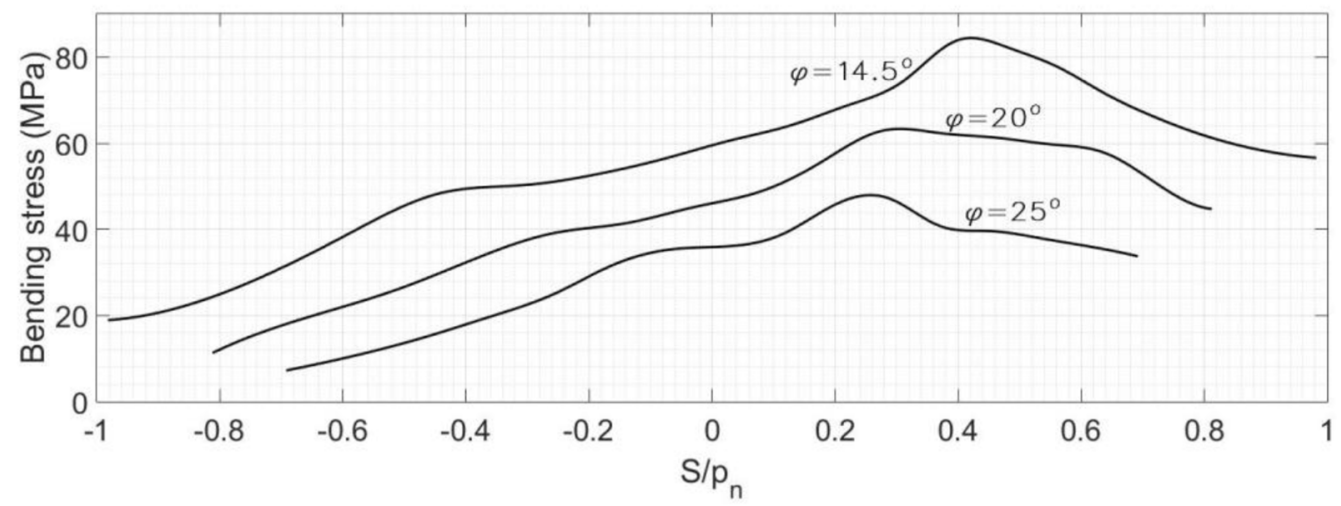

(c)

Fig. 16. Effect of gear parameters on the variation of the bending stress.

maximum stress is always located at a distance $S_{c}$ to the right of point $P$, approximately, corresponding to

$$
S_{c}=0.5 S_{2}
$$

Note that the distance $S_{2}$ can be expressed by

$$
S_{2}=R_{b 1} \psi
$$

where $\psi=\tan \theta-\tan \varphi, \theta=\cos ^{-1} \frac{R_{b 1}}{R_{O 1}}$, and $\varphi$ is the pressure angle.

In the case of low torques corresponding to a real contact ratio less than 2, we can see, in Figure 17, that the stress curve has a discontinuity between $S=-0.19 p_{n}$ and
$S=0.04 p_{n}$. This is due to the fact that in this area the number $n_{1}$ of teeth that are initially in contact goes down from 2 to 1 and then back up to 2 for $S$ greater than $0.04 p_{n}$. This causes a sudden decrease in the overall stiffness of the gear pair and, consequently, a sudden increase in bending stress in this area attaining its maximum at $S_{c}=0.05 S_{2}$, approximately.

Consequently, the critical tooth-root bending stress for a pair of plastics gears may be computed from

$$
\sigma_{F}=\frac{6 \lambda\left(\varepsilon_{r}, S_{c}\right) W_{t o t} \cos \alpha_{F}\left(S_{c}\right) h_{F}\left(S_{c}\right)}{F S_{F}^{2}}
$$




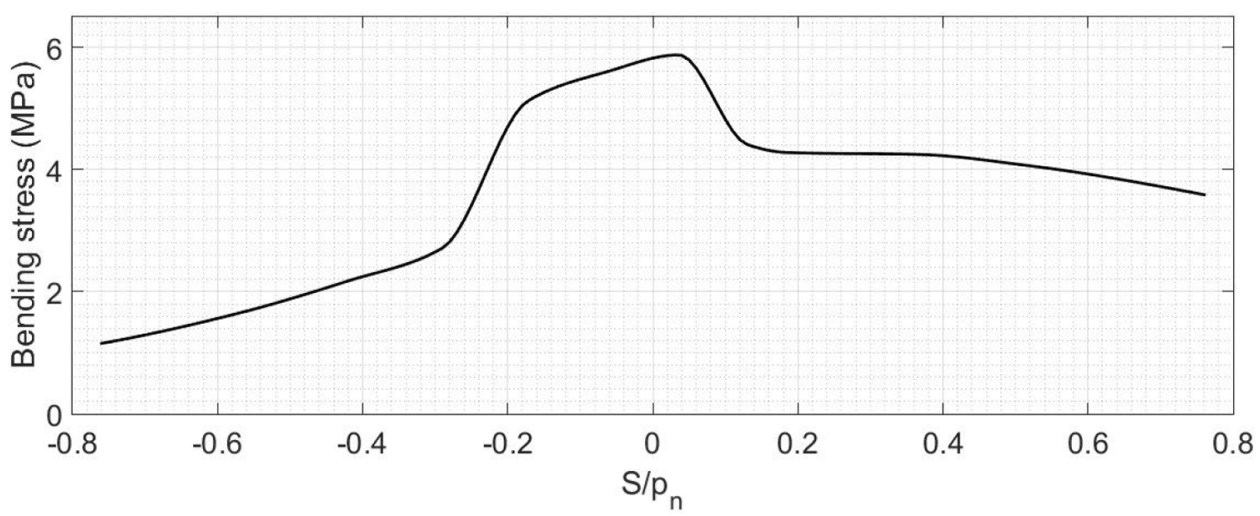

Fig. 17. Variation of the bending stress at the tooth fillet for the $40 / 40$ gear pair under low torque.

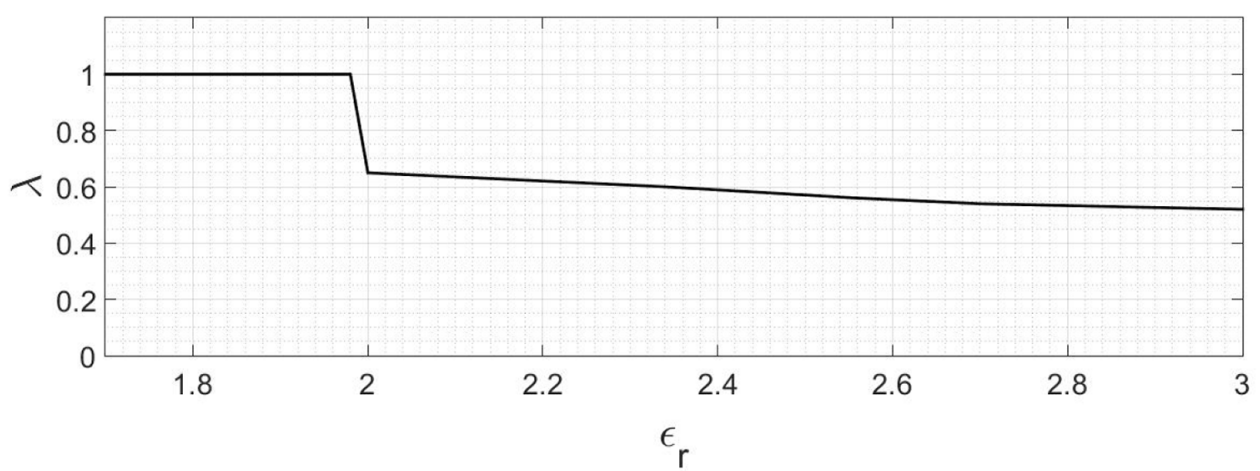

Fig. 18. Variation of $\lambda$ (at point $S_{c}$ ), as a function of $\varepsilon_{\mathrm{r}}$ for the $25 / 25$ Nylon 6 gear pair.

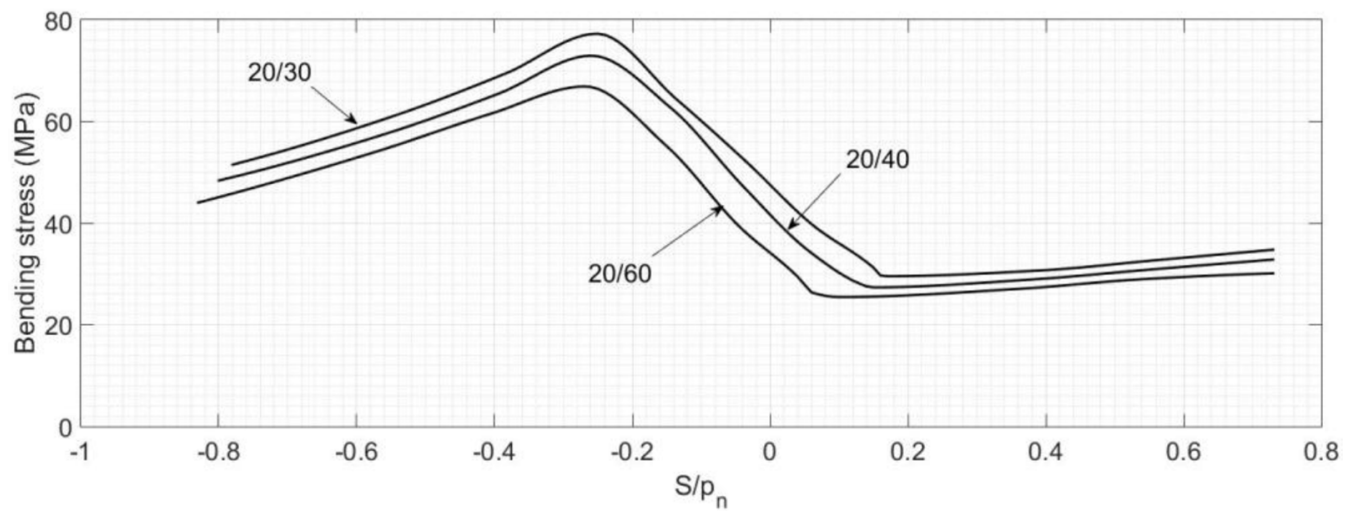

Fig. 19. Variation of the bending stress at the tooth fillet for the $20 / 30,20 / 40$ and $20 / 60$ plastic/steel gear pairs.

where $\lambda\left(\varepsilon_{r}, S_{c}\right)$ is the ratio of the applied load $W_{t r_{c}}$, at the point of the critical bending stress, to the total applied load.

$$
\lambda\left(\varepsilon_{r}, S_{c}\right)=\frac{W_{t r_{\mathrm{c}}}}{W_{t o t}}
$$

Equation (8) is ascertained in Figure 15a and b which shoe the load sharing distributions, where the value of $\lambda$ depends on the position of the point of contact on the line of action and on the real contact ratio. It is, therefore, necessary to establish for each material the graph which shows the variation of $\lambda$ as a function of the real contact ratio. Figure 18 shows this variation of $\lambda$ for the $25 / 25$ Nylon 66 gear pair determined by the proposed method.

\subsubsection{Case of plastic/steel spur gears}

To show the distribution of bending stress along the line of contact of a pair of plastic/steel gears, firstly, we consider the cases of $20 / 30,20 / 40$ and $20 / 60$ gears ratios having a module of $4 \mathrm{~mm}$, a pressure angle of $20^{\circ}$ and a face width of $30 \mathrm{~mm}$. The applied torque is $600 \mathrm{~N} \mathrm{~m}$. The distributions of the tooth-root stress are shown in Figure 19. 
From Figure 19, it can be noticed that the shape of the bending stress distribution in plastic/steel gear pair, is different from the bending stress distribution in the case where both gears are made of plastic. Note also, from Figure 19, that the maximum bending stress is located approximately at a distance $S_{c}^{\prime}=0.3 S_{1}$ to the left of the primitive point $P$ where $S_{1}$ is the distance from the start point of contact on the outer radius of the gear and the point $P$ (point A in Fig. 1). As in the previous case, very similar results were obtained from a study with a lot of combinations of gear parameters pointing to the maximum stress being located at the distance $S_{c}{ }_{c}$ to the left of $P$. The behavior of the plastic/steel gear pairs shown in Figure A.1 may be explained by the fact that compliance $w$ is almost equal to the compliance of the plastic gear as shown in Figure 5 . The minimum value of the compliance is in the area to the left of the point $P(S<0)$. Therefore, the maximum value of the applied load as well as the bending stress, as can be seen in Figure 15b, tend to be located in this area where the compliance is minimum.

The distance $S_{1}$ can be expressed as

$$
S_{1}=R_{b 1} \psi
$$

where $\psi=\tan \theta-\tan \varphi, \theta=\cos ^{-1} \frac{R_{b 1}}{R_{A 1}}$, and $\varphi$ is the pressure angle.

In the case of low torques corresponding to real contact ratios close to the theoretical contact ratio, the behavior of the gear pair is similar to that presented in Figure 17. This can be explained, similarly, to the case of plastic gears and $S_{c}{ }_{c}$ becomes equal to $0.05 S_{2}$, in this case. It can, therefore, be seen that the use of equations (7) and (8) is always possible if we replace $S_{c}$ by $S_{c}^{\prime}$ after having determined $\lambda$ as a function of $\varepsilon_{r}$.

\section{Conclusion}

In this paper, we present a method which predicts the behavior of a pair of plastic/plastic and plastic/steel spur gears. This method enables the calculation of the contact ratio and the bending stress at the tooth root. The results obtained from this method have been further confirmed by finite element calculations.

The modeling of the gear pair during the mashing cycle of a line of contact leads to the calculation of the real contact ratio and to the determination of the maximum bending stress. A parametric study, by varying different parameters such as the number of teeth, pressure angle, and module, yields the effect of these parameters on the contact ratio and the bending stress. In addition, the modeling of the pair of gears in contact during a meshing cycle leads to the following results:

For plastic/plastic spur gears the critical tooth-root stress is obtained when the point of contact is located at a distance $S_{c}=0.5 S_{2}$ and at a distance $S_{c}=0.05 S_{2}$ from the primitive point $P$ when the contact ratio is close to the theoretical contact ratio. Whereas, in the case of plastic/ steel spur gears, the distribution of bending stress during the meshing cycle is different from that of plastic gears. The critical tooth-root stress, in this case, is obtained when the point of contact is located at a distance $S_{c}{ }_{c}=0.3 S_{1}$ and at a distance $S_{c}{ }_{c}=0.05 S_{2}$ from the primitive point $P$ when the contact ratio is close to the theoretical contact ratio.

These results can be used for preliminary designs of plastic/plastic and plastic/steel spur gears. Additionally, they can be incorporated in a proposal for ISO 6336 related to stress calculation of plastic spur gears.

\section{Notation}

$F \quad$ Face width of the gear

$m \quad$ Module

$Z \quad$ Number of teeth

$p_{b} \quad$ Base pitch on base circle

$p_{n} \quad$ Normal base pitch

$R_{b} \quad$ Radius of the base cylinder

$R \quad$ Radius of the pitch circle

$R_{O} \quad$ Radius of the addendum cylinder

$R_{A} \quad$ Radius of the lowest point of contact

$\varphi \quad$ Pressure angle

$W_{\text {tr }} \quad$ Transverse load

Subscripts 1, 2 Designate, respectively, the pinion and the gear

\section{Appendix}

Referring to Figure A.1, let $\mathrm{S}$ be the distance from the point of contact to the primitive point of tangency, $P$, between the pitch circles of the pinion and of the gear. We, then, have

$$
S=|X-x|
$$

with

and

$$
X=R_{b} \tan \theta
$$

$$
x=R_{b} \tan \varphi
$$

Let

$S=R_{b}|\tan \theta-\tan \varphi|=R_{b}|\tan \theta-\tan \varphi+\theta-\theta+\varphi-\varphi|$

However, $\operatorname{inv} \theta=\tan \theta-\theta$ and $\operatorname{inv} \varphi=\tan \varphi-\varphi$, from where

$$
S=R_{b}|\theta-\varphi+\operatorname{inv} \theta-\operatorname{inv} \varphi|
$$

From Figure A.1, we have

$$
\psi=\theta-\varphi+i n v \theta-i n v \varphi
$$

Therefore, we get

$$
S=R_{b} \psi
$$




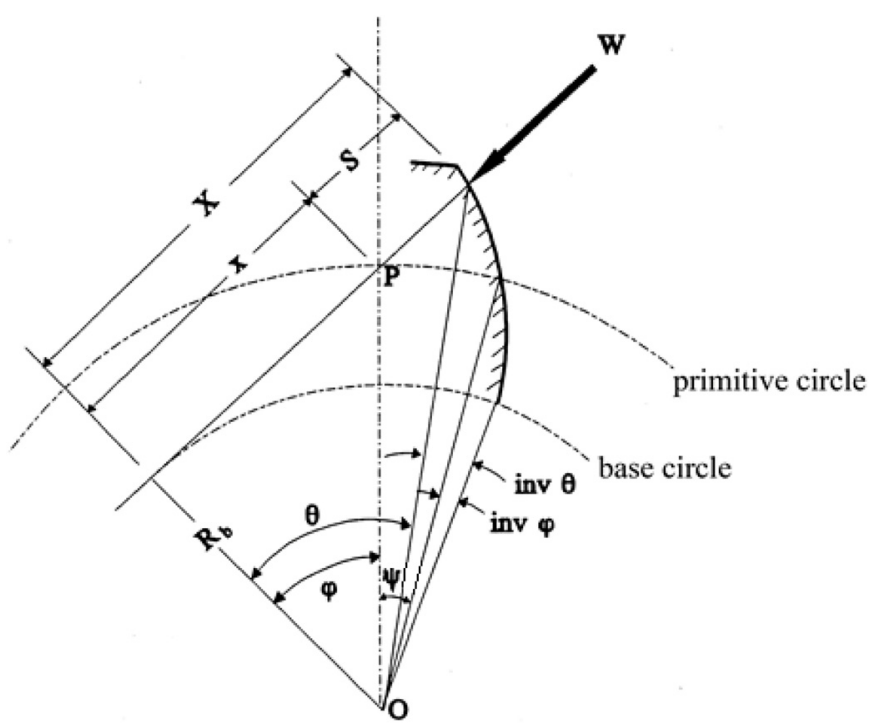

Fig. A.1. Definition of the distance S from the point of contact to the primitive point $P$.

Each point of contact can be characterized by its position $S$ in the plane of action relative to the primitive point $P$. This position can be calculated knowing the angle $\Delta \psi$ between the center line and the point of intersection of the profile of the tooth with the pitch circle of the gear.

Let us start with the case where the head of the gear tooth enters in contact prematurely with the root of the pinion tooth. Figure A.2 shows a pinion meshing with a gear. The angle that the pinion must rotate through so that the tooth $\mathrm{a}_{1}$ comes into contact with the tooth $\mathrm{a}_{2}$, is equal to $\left(\beta_{1}+\Omega_{1}-\gamma_{1}\right)$.

The position of the tooth $\mathrm{a}_{1}$ with respect to the point $\mathrm{P}$ before rotation is equal to

$$
S_{1}=R_{b 1}\left(\beta_{1}+\beta_{0 f}\right)
$$

After rotation, its position is

$$
S_{1}^{\prime}=R_{b 1}\left(\gamma_{1}-\Omega_{1}+\beta_{0 f}\right)
$$

The distance that the tooth $\mathrm{a}_{1}$ must travel along the line of action to come into contact with the tooth $a_{2}$, is equal to

$$
\Delta S=R_{b 1}\left(\beta_{1}+\Omega_{1}-\gamma_{1}\right)
$$

Similar to the case of the pinion, the position of the tooth $\left(a_{2}\right)$ of the gear, measured on the line of action, is equal to (see Fig. A.2)

$$
S_{2}=R_{b 2}\left(\delta_{2}+\delta_{02}\right)
$$

We have, from Figure A.2,

and

$$
\beta_{0 f}=i n v \varphi
$$

$$
\delta_{02}=i n v \varphi_{2}-\beta_{0 f}
$$

where $\varphi_{2}=\cos ^{-}{ }^{1} \frac{R_{b 2}}{R_{o 2}}$ and $R_{b 2}, R_{O 2}$ are, respectively, the base and the outer radii of the gear.

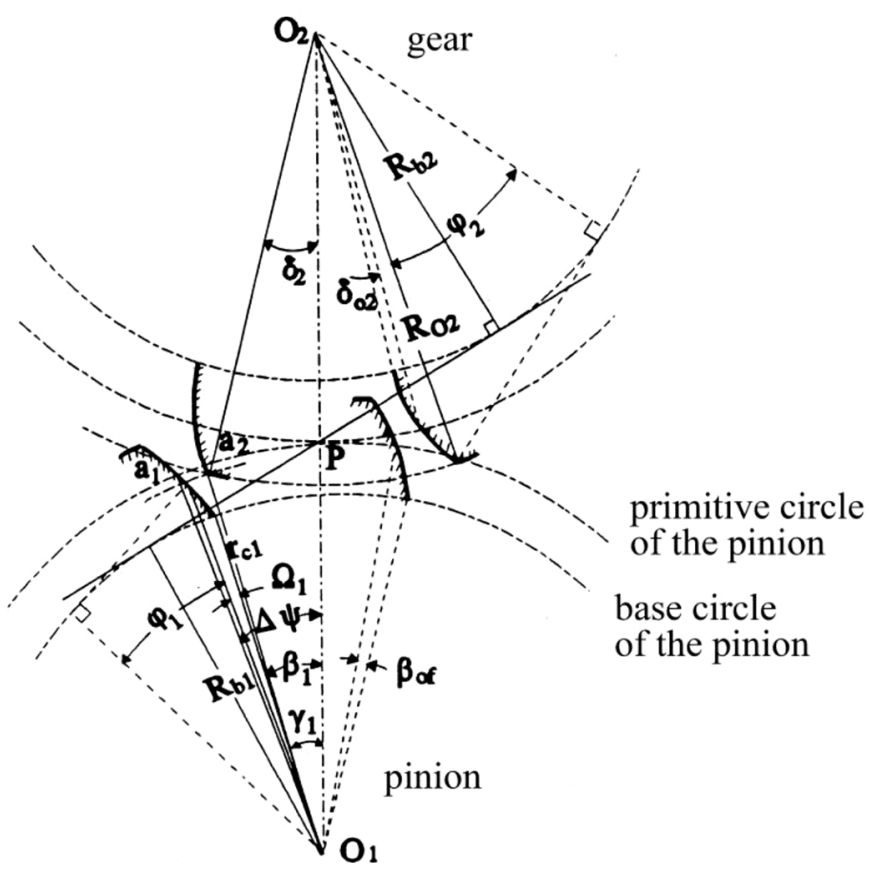

Fig. A.2. Radius $r_{\mathrm{c} 1}$ resulting from premature contact of a pinion tooth with the tip of a gear tooth.

The angle $\beta_{1}$ can be calculated from equations (A.1) and (A.2), which give

$$
\beta_{1}=\psi-\beta_{0 f}
$$

Now under non-slip rolling conditions, the following relationship holds $S_{1}=S_{2}$

When the values of $S_{1}$ and $S_{2}$ are substituted in equations (A.2) and (A.5), we get

$$
R_{b 1}\left(\beta_{1}+\beta_{0 f}\right)=R_{b 2}\left(\delta_{2}+\delta_{02}\right)
$$

The above equation gives

$$
\delta_{2}=\left(\beta_{1}+\beta_{0 f}\right) \frac{Z_{1}}{Z_{2}}-\delta_{02}
$$

We also have, from Figure A.2

and

$$
\frac{r_{c 1}}{\sin \delta_{2}}=\frac{R_{O 2}}{\sin \gamma_{1}}
$$

$$
R_{1}+R_{2}=r_{c 1} \cos \gamma_{1}+R_{O 2} \cos \delta_{2}
$$

The above two equations give

$$
\tan \gamma_{1}=\frac{R_{O 2} \sin \delta_{2}}{R_{1}+R_{2}-R_{O 2} \cos \delta_{2}}
$$

We can then write from Figure A.2

$$
\varphi_{1}=\cos ^{-1} \frac{R_{b 1}}{r_{c 1}}
$$




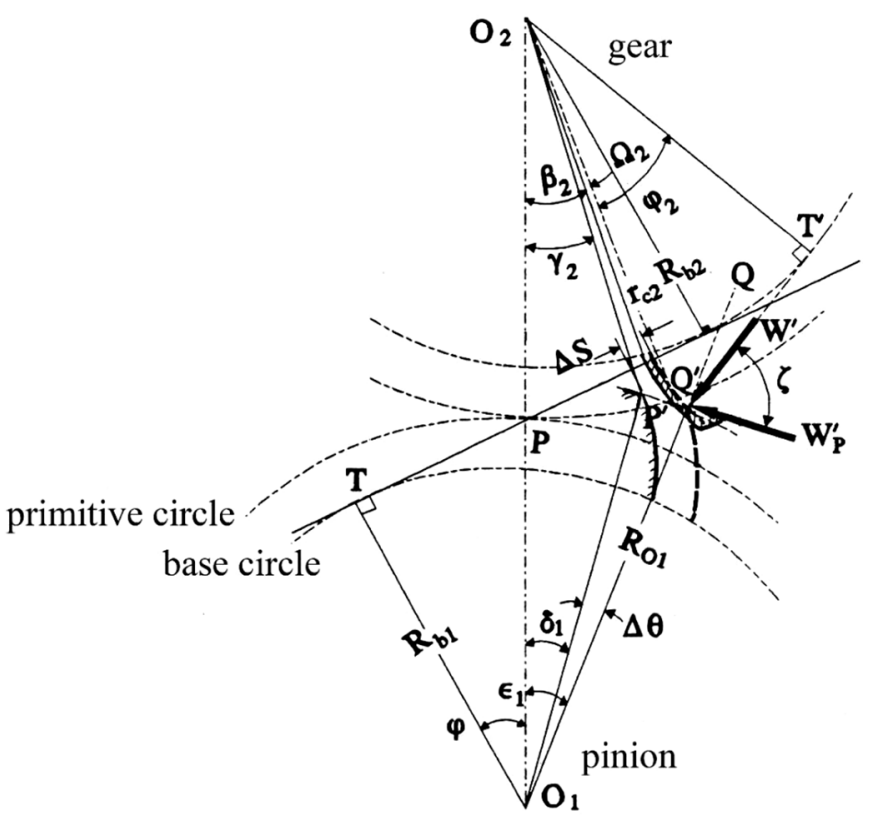

Fig. A.3. Case where the head of the pinion tooth enters in contact prematurely with the root of the gear tooth.

and

$$
\Omega_{1}=\operatorname{inv} \varphi_{1}
$$

From the values of $\beta_{1}, \Omega_{1}$ and $\gamma_{1}$ calculated from equations (A.6), (A.7) and (A.9), we can calculate the value of $\Delta S$ using equation (A.4).

In the case where the head of the pinion tooth enters in contact prematurely with the root of the gear tooth, the treatment is analogous to the foregoing, except that, in this case, index 2, characterizing the gear, will be replaced by index 1 , characterizing the pinion.

\section{Calculation of $R_{b 1}^{\prime}$}

Let us assume that a pair of teeth are in contact enter in contact, prematurely, due to the applied torque on the pinion. Let $W$ be the resulting load on the teeth (see Fig. A.3). The resisting torque about the pinion center, $T$, is given by

$$
T^{\prime}=W^{\prime} R_{b 1}^{\prime}
$$

Two cases arise:

(1) If the pinion tooth is contacted at the root, the value of $R_{b 1}^{\prime}$ would be set equal to $R b_{1}$ because the direction of the load is parallel to the tangent of the base circle of the pinion (see Fig. A.2). We, consequently, get

$$
R_{b 1}^{\prime}=R_{b 1}
$$

(2) If the pinion tooth is contacted at its top, the torque W' (see Fig. A.3) is given by

$$
T^{\prime}=W_{p}^{\prime} R_{O 1}
$$

where

$$
W_{p}^{\prime}=W^{\prime} \cos \zeta
$$

Further, in triangle $\mathrm{O}_{2} \mathrm{Q}^{\prime} \mathrm{T}^{\prime}$, we have

$$
\overline{O_{2} Q^{\prime} T^{\prime}}=\frac{\pi}{2}-\varphi_{2}
$$

Also

$$
\overline{O_{2} Q^{\prime} Q}=\varepsilon_{1}+\beta_{2}+\Omega_{2}
$$

and

$$
\overline{Q Q^{\prime} W^{\prime}}=\frac{\pi}{2}-\zeta
$$

However,

$$
\overline{\mathrm{O}_{2} Q^{\prime} T^{\prime}}=\overline{\mathrm{O}_{2} Q^{\prime} Q}+\overline{Q Q^{\prime} W^{\prime}}
$$

Substituting $\overline{\mathrm{O}_{2} Q^{\prime} T^{\prime}}, \overline{\mathrm{O}_{2} Q^{\prime} Q}$, and $\overline{Q Q^{\prime} W^{\prime}}$ from equations (A.11)-(A.13) into equation (A.14), we get

$$
\frac{\pi}{2}-\varphi_{2}=\varepsilon_{1}+\beta_{2}+\Omega_{2}+\frac{\pi}{2}-\zeta
$$

From where we get

$$
\zeta=\varepsilon_{1}+\beta_{2}+\Omega_{2}+\varphi_{2}
$$

where

$$
\begin{gathered}
\varphi_{2}=\cos ^{-1} \frac{R_{b 2}}{r_{c 2}} \\
\Omega_{2}=\operatorname{inv} \varphi_{2}
\end{gathered}
$$

and

$$
\varepsilon_{1}=\sin ^{-1}\left(\frac{r_{c 2} \sin \left(\beta_{2}+\Omega_{2}\right.}{R_{O 1}}\right)
$$

Then the torque $T$ would be given by

$$
T^{\prime}=R_{O 1} \cos \left(\varepsilon_{1}+\beta_{2}+\Omega_{2}+\varphi_{2}\right) W^{\prime}
$$

From equations (A.10) and (A.15), we finally have

$$
R_{b 1}^{\prime}=R_{O 1} \cos \left(\varepsilon_{1}+\beta_{2}+\Omega_{2}+\varphi_{2}\right)
$$

\section{References}

[1] ISO 6336:2006. Calculation of load capacity of spur and helical gears.

[2] K.W. Hall, H.H. Alvord, Progress reports on evaluation of Zytel and derlin as gears materials. Report, Engineering Research Institute, USA, 1968 
[3] E.A. Cornelius, I.W. Budich, Investigations of gears of acetal resins, J. Konstruktion 22, 103-116 (1970)

[4] H. Yelle, Design of thermoplastic gears with an involute tooth profile, Ph. D. Thesis, University of Waterloo, CA, 1977

[5] M.H. Tsai, Y.C. Tsai, A method for calculating static transmission errors of plastic spur gears using FEM evaluation, J. Finite Elem. Anal. Des. 27, 345-357 (1997)

[6] H.G. Van Melick, Tooth-bending effects in plastic spur gears, J. Gear Technol. 24, 58-66 (2007)

[7] E. Letzelter, J.P. De Vaujany, M. Guingand, L. Chazeau, Quasi-static load sharing model in the case of Nylon 6/6 cylindrical gears, J. Mater. Des. 30, 4360-4368 (2009)

[8] K. Kobayashi, N. Tsukamoto, Formula for dynamic behaviors of tooth deflection and tooth root train in plastic gears, J. Theor. Appl. Mech. 49, 49-56 (2000)

[9] M. Karimpour, K.D. Dearn, D. Walton, A kinematic analysis of meshing polymer gear teeth, Proc. Inst. Mech. Eng. L 224, 101-115 (2010)

[10] E. Letzelter, J.P. Vaujany, M. Guingand, Load-sharing model for polymer cylindrical gears, J. Gear Technol. 28, 2834 (2011)

[11] J. Cathelin, E. Letzelter, M. Guingand et al., Experimental and numerical study of a loaded cylindrical PA66 gear, J. Mech. Des. 135, 041007 (2013)

[12] S. Trobentar, S. Glodež, B. Zafošnik, Deflection analysis of spur polymer gear teeth, J. Multidiscipl. Eng. Sci. Technol. 2, 847-852 (2015)
[13] C. Hasl, H. Liu, P. Oster et al., Method for calculating the tooth root stress of plastic spur gears meshing with steel gears under consideration of deflection-induced load sharing, J. Mech. Mach. Theory 111, 152-163 (2017)

[14] S. Ramasamy, Tooth-bending effects in plastic spur gears influence on load sharing, stresses and wear, J. Sci. Eng. Res. 3, 192-199 (2012)

[15] V. Atanasiu, I. Doroftei, M.R. Iacob, D. Leohchi, Nonlinear dynamics of steel/plastic gears of servomechanism structures, J. Mater. Plastice 48, 98-103 (2011)

[16] T. Jabbour, G. Asmar, Tooth stress calculation of metal spur and helical gears, Mech. Mach. Theory 92, 375-390 (2015)

[17] A. Timoshenko, N.V. Baud, Strength of gear teeth, J. Mech. Eng. 48, 1-5 (1926)

[18] N.L. Pederson, M.F. Jørgensen, On gear tooth stiffness evaluation Niels Leergaard Pedersen, Martin Felix Jørgensen, Comput. Struct. 135, 109-117 (2014)

[19] Y. Yang, J. Wang, Q. Zhou, Y. Huang, J. Zhu, W. Yang, Mesh stiffness modeling considering actual tooth profile geometry for a spur gear pair, Mech. Ind. 19, 306 (2018)

[20] J. Hedlund, A. Lehtovaara, A parameterized numerical model for the evaluation of gear mesh stiffness variation of a helical gear pair, Proc. Inst. Mech. Eng. Part C 222, 1321-1327 (2008)

[21] R.W. Furrow, H.H. Mabie, The measurement of static deflection in spur gear teeth, J. Mech. 5, 147-150 (1970)

[22] N.G. Mcrum, C.P. Buckley, C.B. Bucknall, Principles of polymer engineering, Oxford Science Publications, Canada, 1990, 204

Cite this article as: T. Jabbour, G. Asmar, M. Abdulwahab, J. Nasr, Real contact ratio and tooth bending stress calculation for plastic/plastic and plastic/steel spur gears, Mechanics \& Industry 22, 30 (2021) 